\title{
Troisième groupe de cohomologie non ramifiée des torseurs universels sur les surfaces rationnelles
}

\author{
Yang Cao
}

\begin{abstract}
Résumé. Soit $k$ un corps de caractéristique zéro. Soit $X$ une $k$-surface projective et lisse géométriquement rationnelle. Soit $\mathcal{T}$ un torseur universel sur $X$ possédant un $k$-point, et soit $\mathcal{T}^{c}$ une compactification lisse de $\mathcal{T}$. La question de savoir si $\mathcal{T}^{c}$ est $k$-birationnel à un espace projectif est encore ouverte. On sait que les deux premiers groupes de cohomologie non ramifiée de $\mathcal{T}$ et $\mathcal{T}^{\mathcal{C}}$ sont réduits à leur partie constante. On donne une condition suffisante en termes de la structure galoisienne du groupe de Picard géométrique de $X$ assurant l'énoncé analogue pour les troisièmes groupes de cohomologie non ramifiée de $\mathcal{T}$ et $\mathcal{T}^{c}$. Ceci permet de montrer que $H_{\mathrm{nr}}^{3}\left(\mathcal{T}^{c}, \mathbb{Q} / \mathbb{Z}(2)\right) / H^{3}(k, \mathbb{Q} / \mathbb{Z}(2))$ est nul si $X$ est une surface de Châtelet généralisée, et que ce groupe est réduit à sa partie 2-primaire si $X$ est une surface de del Pezzo de degré au moins 2.
\end{abstract}

\section{[English]}

Title. Third unramified cohomology group of universal torsors on rational surfaces

Abstract. Let $k$ a field of characteristic zero. Let $X$ be a smooth, projective, geometrically rational $k$-surface. Let $\mathcal{T}$ be a universal torsor over $X$ with a $k$-point et $\mathcal{T}^{c}$ a smooth compactification of $\mathcal{T}$. There is an open question : is $\mathcal{T}^{c} k$-birationally equivalent to a projective space? We know that the unramified cohomology groups of degree 1 and 2 of $\mathcal{T}$ and $\mathcal{T}^{c}$ are reduced to their constant part. For the analogue of the third cohomology groups, we give a sufficient condition using the Galois structure of the geometrical Picard group of $X$. This enables us to show that $H_{\mathrm{nr}}^{3}\left(\mathcal{T}^{c}, \mathbb{Q} / \mathbb{Z}(2)\right) / H^{3}(k, \mathbb{Q} / \mathbb{Z}(2))$ vanishes if $X$ is a generalised Châtelet surface and that this group is reduced to its 2-primary part if $X$ is a del Pezzo surface of degree at least 2.

Keywords. Rationality question; unramified cohomology; universal torsor

2010 Mathematics Subject Classification. 14E08, 12G05

Received by the Editors on May 3, 2017, and in final form on July 18, 2018.

Accepted on October 16, 2018.

Yang Cao

Laboratoire de Mathématiques d'Orsay, Université Paris-Sud, CNRS, Université Paris-Saclay, 91405 Orsay, France

e-mail: yangcao1988@gmail.com 


\section{Table des matières}

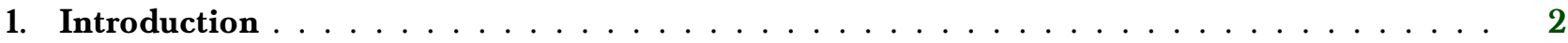

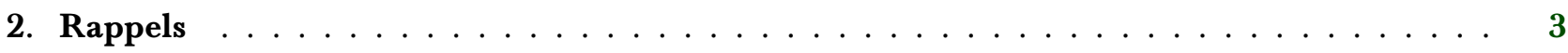

3. Cohomologie motivique à coefficients $\mathbb{Z}(2)$ d'un torseur sous un tore $\ldots \ldots \ldots$

4. Cohomologie motivique à coefficients $\mathbb{Z}(2)$ des torseurs universels sur une surface géométri-

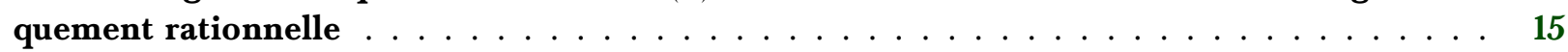

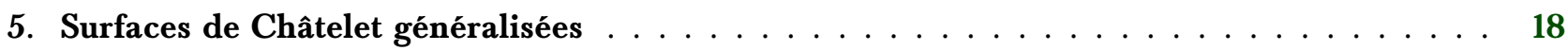

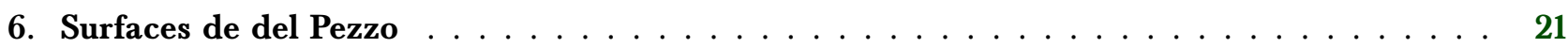

\section{Introduction}

Soit $k$ un corps de caractéristique 0 . Pour une variété $X$ sur $k$ et un faisceau étale $F$ sur $X$, la cohomologie non ramifiée de $X$ de degré $n$ est ici par définition le groupe

$$
H_{\mathrm{nr}}^{n}(X, F):=H_{\text {Zariski }}^{0}\left(X, \mathcal{H}^{n}(X, F)\right),
$$

où $\mathcal{H}^{n}(X, F)$ est le faisceau Zariski associé au préfaisceau $\{U \subset X\} \mapsto H_{e ̂ t}^{n}(U, F)$. Ces groupes sont des invariants $k$-birationnels de $k$-variétés intègres projectives et lisses ([CT95, Thm. 4.1.1]). Si $X$ est projective, lisse et $k$-rationnelle, par [CT95, Thm. 4.1.1 et Prop. 4.1.4], on a $H^{i}(k, \mathbb{Q} / \mathbb{Z}(j)) \stackrel{\sim}{\rightarrow} H_{\mathrm{nr}}^{i}(X, \mathbb{Q} / \mathbb{Z}(j))$ pour tous entiers $i \in \mathbb{N}, j \in \mathbb{Z}$.

Soit $X$ une $k$-surface projective, lisse, géométriquement rationnelle. Soit $\mathcal{T} \rightarrow X$ un torseur universel sur $X$ [CTS87]. Ce torseur est une $k$-variété géométriquement rationnelle (Corollaire 2.9). En 1979, ColliotThélène et Sansuc [CTS80, Question Q1, p. 227] ont posé la question : si $\mathcal{T}$ possède un point rationnel, la $k$-variété $\mathcal{T}$ est-elle une $k$-variété $k$-rationnelle? Cette question est toujours ouverte.

Un certain nombre d'invariants $k$-birationnels sont triviaux sur toute compactification lisse $\mathcal{T}^{c}$ de $\mathcal{T}$. Ainsi les applications de restriction $H^{i}(k, \mathbb{Q} / \mathbb{Z}(i-1)) \rightarrow H_{\mathrm{nr}}^{i}\left(\mathcal{T}^{c}, \mathbb{Q} / \mathbb{Z}(i-1)\right)$ sont des isomorphismes pour $i=1$ et $i=2$. Le cas $i=1$ est facile. Dans le cas $i=2$, ceci dit que l'application de restriction $\operatorname{Br}(k) \rightarrow \operatorname{Br}\left(\mathcal{T}^{c}\right)$ sur les groupes de Brauer est un isomorphisme. Pour ce résultat, voir [CTS77, Thm. 1], [CTS87, Thm. 2.1.2], [HS, Prop. 1.8] et le Théorème 2.8 ci-dessous. Par ailleurs, pour $\mathcal{T}$ possédant un $k$ point, on sait (Proposition 2.12) que pour tous $i \in \mathbb{N}, j \in \mathbb{Z}$, l'image de $H_{\mathrm{nr}}^{i}(X, \mathbb{Q} / \mathbb{Z}(j))$ dans $H_{\mathrm{nr}}^{i}(\mathcal{T}, \mathbb{Q} / \mathbb{Z}(j))$ est réduite à $H^{i}(k, \mathbb{Q} / \mathbb{Z}(j))$.

Dans le présent article, pour $X, \mathcal{T}$ et $\mathcal{T}^{c}$ comme ci-dessus, nous étudions les groupes

$$
H_{\mathrm{nr}}^{3}\left(\mathcal{T}^{c}, \mathbb{Q} / \mathbb{Z}(2)\right) / H^{3}(k, \mathbb{Q} / \mathbb{Z}(2)) \subset H_{\mathrm{nr}}^{3}(\mathcal{T}, \mathbb{Q} / \mathbb{Z}(2)) / H^{3}(k, \mathbb{Q} / \mathbb{Z}(2)) .
$$

Dans [Cao], nous avons établi que $H_{\mathrm{nr}}^{3}\left(\mathcal{T}^{c}, \mathbb{Q} / \mathbb{Z}(2)\right) / H^{3}(k, \mathbb{Q} / \mathbb{Z}(2))$ est fini.

Les principaux résultats du présent article sont les suivants.

(a) Le quotient $H_{\mathrm{nr}}^{3}(\mathcal{T}, \mathbb{Q} / \mathbb{Z}(2)) / H^{3}(k, \mathbb{Q} / \mathbb{Z}(2))$ est fini.

(b) $\operatorname{Si~} H^{1}\left(k, \operatorname{Sym}^{2} \operatorname{Pic}\left(X_{\bar{k}}\right)\right)=0$ et $X(k) \neq \emptyset$, alors $\frac{H_{\mathrm{nr}}^{3}(\mathcal{T}, \mathbb{Q} / \mathbb{Z}(2))}{H^{3}(k, \mathbb{Q} / \mathbb{Z}(2))}=0$ (Théorème 4.7). Pour établir ce résultat, nous appliquons aux torseurs sous un tore une technique développée par A. Merkurjev [Me16] pour étudier les torseurs sous un groupe semisimple.

(c) Si $X$ est une surface de Châtelet généralisée, c'est-à-dire un fibré en coniques sur $\mathbb{P}_{k}^{1}$ possédant une section sur une extension quadratique de $k$, et $X(k) \neq \emptyset$, alors $\frac{H_{\mathrm{nr}}^{3}\left(\mathcal{T}^{c}, \mathbb{Q} / \mathbb{Z}(2)\right)}{H^{3}(k, \mathbb{Q} / \mathbb{Z}(2))}$ est nul (Théorème 5.1).

(d) Si $X$ est une surface projective, lisse, $k$-birationnellement équivalente à une surface de del Pezzo de degré $\geq 2$ ou à une surface fibrée en coniques au-dessus d'une conique, alors $\frac{H_{\mathrm{nr}}^{3}\left(\mathcal{T}^{c}, \mathbb{Q} / \mathbb{Z}(2)\right)}{H^{3}(k, \mathbb{Q} / \mathbb{Z}(2))}$ est purement 2-primaire (Théorème 6.7). 


\section{Conventions et notations}

Soit $k$ un corps quelconque de caractéristique 0 . On note $\bar{k}$ une clôture algébrique et $\Gamma_{k}:=\operatorname{Gal}(\bar{k} / k)$.

Une $k$-variété $X$ est un $k$-schéma séparé de type fini. Pour $X$ une telle variété, on note $k[X]$ son anneau des fonctions globales, $k[X]^{\times}$son groupe des fonctions inversibles, et $\operatorname{Pic}(X):=H_{\text {êt }}^{1}\left(X, \mathbb{G}_{m}\right)$ son groupe de Picard. Si $X$ est lisse, on note $\operatorname{Br}(X):=H_{\text {êt }}^{2}\left(X, \mathbb{G}_{m}\right)$ son groupe de Brauer, $\mathrm{CH}^{i}(X)$ son groupe de Chow de codimension $i$ et $\mathrm{CH}_{i}(X)$ son groupe de Chow de dimension $i$. Pour $X / k$ projective lisse, notons $A_{0}(X) \subset \mathrm{CH}_{0}(X)$ le groupe de classes des 0 -cycles de degré 0 . Pour tous $i \in \mathbb{N}, j \in \mathbb{Z}$, on note $\frac{H_{\mathrm{nr}}^{i}(X, \mathbb{Q} / \mathbb{Z}(j))}{H^{i}(k, \mathbb{Q} / \mathbb{Z}(j))}$ le conoyau du morphisme $H^{i}(k, \mathbb{Q} / \mathbb{Z}(j)) \rightarrow H_{\mathrm{nr}}^{i}(X, \mathbb{Q} / \mathbb{Z}(j))$.

Tous les groupes de cohomologie ou d'hypercohomologie utilisés dans cet article sont des groupes de cohomologie étale, sauf les groupes de cohomologie de Zariski $H^{i}\left(X, \mathcal{K}_{j}\right)$ à valeurs dans des faisceaux de $K$-théorie algébrique. Pour chaque schéma $X$, notons $D_{e t t}^{+}(X)$ la catégorie dérivée bornée à gauche de la catégorie des faisceaux étales. Pour les propriétés de la catégorie dérivée d'une catégorie abélienne, voir [KS, §13.1]. Pour tout $n \in \mathbb{Z}$, on a la sous-catégorie $D_{e t}^{\geq n}(X) \subset D_{e t}^{+}(X)$ (cf. [KS, Notation 13.1.11]) et les foncteurs de troncature $\tau^{\leq n}$ et $\tau^{\geq n}$ ([KS, Déf. 12.3.1 et Prop. 13.1.5]). Soit

$$
-\otimes^{L}-: D_{e ́ t}^{+}(X) \times D_{e ̂ t}^{+}(X) \rightarrow D_{e ̂ t}^{+}(X):(F, G) \mapsto F \otimes_{\mathbb{Z}}^{L} G .
$$

Puisque la tor-dimension de $\mathbb{Z}$ est 1 , le foncteur ci-dessus est bien défini et on a

$$
-\otimes^{L}-: D_{e ̂ t}^{\geq i}(X) \times D_{e t}^{\geq j}(X) \rightarrow D_{e ́ t}^{\geq i+j-1}(X) .
$$

Soit $T$ un $k$-tore. Notons $T^{*}=\operatorname{Hom}_{\bar{k}-g p}\left(T_{\bar{k}}, \mathbb{G}_{m, \bar{k}}\right)$ le réseau galoisien défini par le groupe des caractères géométriques du tore $T$. Donc $T^{*} \subset \bar{k}[T]^{\times}$.

Pour un groupe abélien $A$ et un entier $n \in \mathbb{Z}$, on note $A[n]:=\{x \in A, n x=0\}$ et $A_{\text {tors }}$ le sous-groupe de torsion de $A$. On note $\operatorname{Sym}^{2} A$ la deuxième puissance symétrique de $A$ ([Bour, III §6]) et $\wedge^{i} A$ la $i$-ième puissance extérieure de $A$ ([Bour, III §7]). Si $A \cong A_{1} \oplus A_{2}$, on a des isomorphismes naturels

$$
\operatorname{Sym}^{2} A_{1} \oplus\left(A_{1} \otimes A_{2}\right) \oplus \operatorname{Sym}^{2} A_{2} \stackrel{\sim}{\rightarrow} \operatorname{Sym}^{2} A \quad \text { et } \quad \wedge^{2} A_{1} \oplus\left(A_{1} \otimes A_{2}\right) \oplus \wedge^{2} A_{2} \stackrel{\sim}{\rightarrow} \wedge^{2} A
$$

Si $A$ est un réseau, on a la suite exacte naturelle

$$
0 \rightarrow \wedge^{2} A \rightarrow A \otimes_{\mathbb{Z}} A \rightarrow \operatorname{Sym}^{2} A \rightarrow 0
$$

où $\wedge^{2} A \rightarrow A \otimes_{\mathbb{Z}} A$ envoie $a \wedge b$ sur $a \otimes b-b \otimes a$.

\section{Rappels}

Dans cette section, on fait des rappels sur plusieurs sujets : cohomologie motivique, variétés toriques, variétés cellulaires, torseurs universels et leur cohomologie à coefficients $\mathbb{G}_{m}$. Soit $k$ un corps de caractéristique 0 .

\section{A. Cohomologie motivique}

Notons $K_{i}(A)$ le $i$-ième groupe de $K$-théorie de Quillen d'un anneau $A$. Sur tout schéma $X$, on note $\mathcal{K}_{i}$ le faisceau pour la topologie de Zariski sur $X$ associé au préfaisceau $U \mapsto K_{i}\left(H^{0}\left(U, \mathcal{O}_{X}\right)\right)$ et $\mathcal{K}_{i \text {,ét }}$ celui pour la topologie étale. On note $H^{i}\left(X, \mathcal{K}_{j}\right)$ ses groupes de cohomologie pour la topologie de Zariski. Par la résolution de Gersten (un théorème de Quillen, cf. [CTHK] pour une démonstration du cas général), si $X$ est une $k$-variété lisse, le groupe $H^{i}\left(X, \mathcal{K}_{j}\right)$ est le $i$-ième groupe de cohomologie du complexe :

$$
0 \rightarrow \oplus_{x \in X^{(0)}} K_{j}(k(x)) \rightarrow \oplus_{x \in X^{(1)}} K_{j-1}(k(x)) \rightarrow \cdots \rightarrow \oplus_{x \in X^{(j)}} \mathbb{Z} \rightarrow 0,
$$

où $X^{(l)}$ est l'ensemble des points de codimension $l$ de $X$ pour tout $l \in \mathbb{Z}_{\geq 0}$. 
On utilise le complexe motivique $\mathbb{Z}(r)$ de faisceaux de cohomologie étale sur les variétés lisses sur $k$ pour $r=0,1,2$, comme défini par Lichtenbaum ([L87] [L90]). On utilise le complexe motivique "de Lichtenbaum" pour pouvoir utiliser la méthode de Merkurjev dans [Me16]. Alors $\mathbb{Z}(0)=\mathbb{Z}, \mathbb{Z}(1) \stackrel{\sim}{\rightarrow} \mathbb{G}_{m}[-1]$, et $\mathbb{Z}(2)$ est supporté en degré 1 et 2 . Si $X$ est un schéma de type fini sur $k$, on a $\mathcal{H}^{2}(X, \mathbb{Z}(2)) \cong \mathcal{K}_{2 \text {,ét }}(X)$ et un triangle dans $D_{e t}^{+}(\mathcal{T})$ pour $r=0,1,2$ :

$$
\mathbb{Z}(r) \stackrel{n}{\longrightarrow} \mathbb{Z}(r) \longrightarrow \mathbb{Z} / n(r) \stackrel{+1}{\longrightarrow} .
$$

De plus, on sait ([Ka93, Ka96, Su], cf. [CT15, §1]) :

Théorème 2.1. Soit $X$ une $k$-variété lisse géométriquement intègre de corps de fonctions $k(X)$.

(i) On a les égalités : $\mathbb{H}^{0}(X, \mathbb{Z}(2))=0, \mathbb{H}^{1}(X, \mathbb{Z}(2))=K_{3, \text { indec }}(k(X)), \mathbb{H}^{2}(X, \mathbb{Z}(2))=H^{0}\left(X, \mathcal{K}_{2}\right)$ et $\mathbb{H}^{3}(X, \mathbb{Z}(2))=H^{1}\left(X, \mathcal{K}_{2}\right)$, où

$$
K_{3, \text { indec }}(k(X)):=\operatorname{Coker}\left(K_{3}^{\text {Milnor }}(k(X)) \rightarrow K_{3}(k(X))\right) .
$$

De plus, $K_{3, \text { indec }}(\bar{k}(X))$ est extension d'un groupe uniquement divisible par $\mathbb{Q} / \mathbb{Z}(2)$.

(ii) On a une suite exacte naturelle:

$$
0 \longrightarrow \mathrm{CH}^{2}(X) \stackrel{\mathrm{cl}}{\longrightarrow} \mathbb{H}^{4}(X, \mathbb{Z}(2)) \longrightarrow H_{\mathrm{nr}}^{3}(X, \mathbb{Q} / \mathbb{Z}(2)) \longrightarrow 0,
$$

où $\mathrm{cl}$ est induit par $\mathrm{CH}^{2}(X) \cong H^{4}\left(X, \mathcal{K}_{2}\right) \cong \mathbb{H}_{\text {Zari }}^{4}(X, \mathbb{Z}(2)) \rightarrow \mathbb{H}^{4}(X, \mathbb{Z}(2))([\operatorname{Ka} 96,(10)])$.

Dans [L87, Prop. 2.5], Lichtenbaum a défini le cup-produit des complexes motiviques

$$
\cup: \mathbb{Z}(1) \otimes^{L} \mathbb{Z}(1) \rightarrow \mathbb{Z}(2),
$$

et ce morphisme induit un morphisme de faisceaux :

$$
\mathbb{G}_{m} \otimes \mathbb{G}_{m} \cong \mathcal{H}^{2}\left(\mathbb{Z}(1) \otimes{ }^{L} \mathbb{Z}(1)\right) \rightarrow \mathcal{H}^{2}(\mathbb{Z}(2)) \cong \mathcal{K}_{2 \text {,ét }}
$$

qui est exactement le morphisme $\mathbb{G}_{m} \otimes \mathbb{G}_{m} \cong \mathcal{K}_{1} \otimes \mathcal{K}_{1} \rightarrow \mathcal{K}_{2 \text {,ét }}$ induit par le cup-produit de groupes de K-théorie $K_{1} \otimes K_{1} \rightarrow K_{2}$ ([L87, Rem. 2.6]). Donc la composition

$$
H^{2}\left(X, \mathbb{Z}_{X}(1)\right) \otimes H^{2}\left(X, \mathbb{Z}_{X}(1)\right) \stackrel{\sim}{\rightarrow} \operatorname{Pic}(X) \otimes \operatorname{Pic}(X) \stackrel{\text { intersection }}{\longrightarrow} \mathrm{CH}^{2}(X) \rightarrow \mathbb{H}^{4}(X, \mathbb{Z}(2))
$$

est exactement le morphisme induit par le cup-produit ci-dessus.

\section{B. Variétés toriques}

Par [Sum, Cor. 2] et [Oda, Thm. 1.10] on a :

Théorème 2.2. Supposons que $k$ est algébriquement clos. Soit $X$ une variété torique lisse sous le tore $\mathbb{G}_{m}^{n}$ sur $k$. Alors pour chaque $\mathbb{G}_{m}^{n}$-orbite $Z$ de codimension $i$, il existe une sous-variété torique ouverte $U \subset X$ telle que $Z \subset U$ et $U \stackrel{\sim}{\rightarrow} \mathbb{G}_{m}^{n-i} \times \mathbb{A}^{i}$ comme variété torique, où l'action de $\mathbb{G}_{m}^{n}$ sur $\mathbb{G}_{m}^{n-i} \times \mathbb{A}^{i}$ est la multiplication.

Comme conséquence, on a :

Corollaire 2.3. Sous les hypothèses du Théorème 2.2, soient $Z_{1}, Z_{2}$ deux $\mathbb{G}_{m}^{n}$-orbites de codimension 1 de $X$. Alors il existe un nombre fini de $\mathbb{G}_{m}^{n}$-orbites de codimension 2 , notons-les $S_{j}$, telles que leurs adhérences schématiques satisfont $\overline{Z_{1}} \cap \overline{Z_{2}}=\bigcup_{j} \overline{S_{j}}$. 


\section{C. Variétés cellulaires}

Définition 2.4. ([Ka97, Définition 3.2]) Une variété $X$ sur $k$ a une décomposition cellulaire (brièvement : est cellulaire) si elle est vide ou s'il existe un sous-ensemble fermé propre $Z \subset X$ tel que $X \backslash Z$ soit isomorphe à un espace affine et $Z$ ait une décomposition cellulaire.

Proposition 2.5. ([Cao, Prop. 2.2]) Soit $k$ un corps algébriquement clos.

(1) Une surface projective, lisse, $k$-rationnelle est cellulaire.

(2) ([Fu93, Lemme, p. 103]) Une variété torique, projective, lisse sur $k$ est cellulaire.

(3) Soient $T$ un tore sur $k$ et $T^{c}$ une $T$-variété torique, projective, lisse. Soient $X$ une variété cellulaire sur $k$ et $Y \rightarrow X$ un $T$-torseur. Alors $Y^{c}:=Y \times^{T} T^{c}$ est cellulaire.

Rappelons que $Y \times^{T} T^{c}$ est le quotient $\left(Y \times T^{c}\right) / T$, où l'action de $T$ est défini par $t \cdot(y, a):=\left(t \cdot y, t^{-1} \cdot a\right)$ pour tous $t \in T, y \in Y$ et $a \in T^{c}$. Ce quotient existe par [PV, Thm. 4.19] et [An73, Appendice 1, Thm. 6].

Par [Fu84, Exemple 19.1.11], on a

Théorème 2.6. Supposons que $k$ est algébriquement clos. Soient $X$ une variété lisse cellulaire sur $k$ et $n$ un entier. Pour tout entier $i$, le groupe $\mathrm{CH}^{i}(X)$ est de type fini et sans torsion, et le morphisme classe de cycle $\mathrm{CH}^{i}(X) \otimes \mathbb{Z} / n \rightarrow H^{2 i}(X, \mathbb{Z} / n)$ est un isomorphisme. Pour tout entier $i$ impair, on a $H^{i}(X, \mathbb{Z} / n)=0$ et donc $H^{i}\left(X, \mathbb{Z}_{l}\right)=0$ pour tout nombre premier $l$.

Soit $X$ une variéte lisse. On a un homomorphisme naturel :

$$
\operatorname{Pic}(X) \otimes k^{\times} \rightarrow H^{1}\left(X, \mathcal{K}_{2}\right) .
$$

En effet, on a le diagramme commutatif suivant

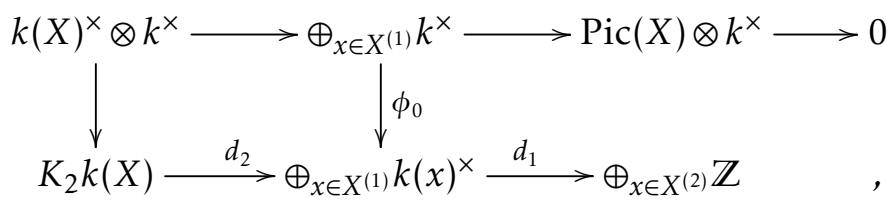

où la première ligne est obtenue à partir de la suite exacte définissant le groupe $\operatorname{Pic}(X)$ par tensorisation avec $k^{\times}$et la deuxière ligne est la résolution de Gersten. On vérifie que la composé $d_{1} \circ \phi_{0}$ vaut zéro, ce qui permet de définir l'homomorphisme (2.6) par chasse au diagramme.

Proposition 2.7. Supposons que $k$ est algébriquement clos. Soit $X$ une variété lisse, connexe, rationnelle sur $k$. Supposons que X est projective ou cellulaire. Alors l'homomorphisme (2.6) est un isomorphisme.

Démonstration. Si $X$ est projective, ceci résulte de A. Pirutka [Pir, Prop. 2.6].

Si $X$ est cellulaire, on fixe une décomposition cellulaire de $X$. Soient $n=\operatorname{dim}(X)$ et $U \subset X$ l'ouvert isomorphe à $\mathbb{A}^{n}$ dans la décomposition cellulaire. Notons $Z:=X \backslash U$. On a $H^{0}\left(U, \mathcal{K}_{2}\right)=K_{2}(k), H^{1}\left(U, \mathcal{K}_{2}\right)=0$ et $\operatorname{Pic}(X) \cong \operatorname{Div}_{Z}(X)$. Par la résolution de Gersten (2.1), on a une suite exacte :

$$
0 \rightarrow H^{0}\left(X, \mathcal{K}_{2}\right) \rightarrow H^{0}\left(U, \mathcal{K}_{2}\right) \rightarrow \operatorname{ker}(\psi) \stackrel{\phi_{1}}{\longrightarrow} H^{1}\left(X, \mathcal{K}_{2}\right) \rightarrow H^{1}\left(U, \mathcal{K}_{2}\right)=0,
$$

où $\psi: \oplus_{x \in Z^{(0)}} k(x)^{\times} \stackrel{\text { div }}{\longrightarrow} \oplus_{x \in Z^{(1)}} \mathbb{Z}$. Alors $H^{0}\left(X, \mathcal{K}_{2}\right) \cong H^{0}\left(U, \mathcal{K}_{2}\right)$ et $\phi_{1}$ est un isomorphisme.

Puisque $\operatorname{div}\left(k^{\times}\right)=0$, on a $\oplus_{x \in Z^{(0)}} k^{\times} \subset \operatorname{ker}(\psi)$. Pour tout $x \in Z^{(0)}$, il existe une unique sous-variété réduite localement fermée $V_{x}$ de codimension 1 dans la décomposition cellulaire telle que $x \in V_{x}, V_{x} \cong$ $\mathbb{A}^{n-1}$ et $V_{x} \cap V_{x^{\prime}}=\emptyset$ pour $x \neq x^{\prime} \in Z^{(0)}$. Donc

$$
k^{\times} \cong \operatorname{ker}\left(k(x)^{\times} \stackrel{\text { div }}{\longrightarrow} \oplus_{y \in V_{x}^{(1)}} \mathbb{Z}\right) \quad \text { et } \quad \operatorname{ker}(\psi) \subset \oplus_{x \in Z^{(0)}} k^{\times} .
$$

Alors $\operatorname{ker}(\psi) \cong \oplus_{x \in Z^{(0)}} k^{\times} \cong \operatorname{Div}_{Z}(X) \otimes k^{\times} \cong \operatorname{Pic}(X) \otimes k^{\times}$. Puisque $\phi_{0}$ et $\phi_{1}$ sont induits par les inclusions $\left\{k^{\times} \subset k(x)^{\times}\right\}_{x \in X^{(1)}}$, on a $\phi_{0}=\phi_{1}$ et le résultat en découle. 


\section{D. Torseurs universels}

Soient $X$ une $k$-variété lisse, $T$ un $k$-tore et $\mathcal{T} \rightarrow X$ un $T$-torseur. La composition $H^{1}(X, T) \rightarrow$ $H^{1}\left(X_{\bar{k}}, T_{\bar{k}}\right) \rightarrow \operatorname{Hom}\left(T^{*}, \operatorname{Pic}\left(X_{\bar{k}}\right)\right)$ associe à $\mathcal{T} \rightarrow X$ un homomorphisme galoisien $T^{*} \stackrel{\text { type }}{\longrightarrow} \operatorname{Pic}\left(X_{\bar{k}}\right)$, appelé le type du torseur. Lorsque le type est un isomorphisme, on dit [CTS87] que $\mathcal{T} \rightarrow X$ est un torseur universel sur $X$.

Théorème 2.8. Soit $X$ une $k$-variété lisse géométriquement intègre, avec $\bar{k}^{\times} \cong \bar{k}[X]^{\times}$et $\operatorname{Pic}\left(X_{\bar{k}}\right)$ de type fini et sans torsion. Soit $\mathcal{T} \rightarrow X$ un torseur universel. Soit $T^{c}$ une $T$-variété torique, projective, lisse. Soit $\mathcal{T}^{c}=\mathcal{T} \times^{T} T^{c}$.

On a les propriétés suivantes :

(i) $[\mathrm{CTS} 87, \mathrm{Thm} .2 .1 .2] \bar{k}^{\times} \cong \bar{k}[\mathcal{T}]^{\times}$.

(ii) $\left[\mathrm{CTS} 87\right.$, Thm. 2.1.2] $\operatorname{Pic}\left(\mathcal{T}_{\bar{k}}\right) \cong 0$.

(iii) [CTS87, Thm. 2.1.2] On a un isomorphisme de $\Gamma_{k}$-modules $\operatorname{Div}_{\mathcal{T}^{c}-\mathcal{T}}\left(\mathcal{T}_{\bar{k}}^{c}\right) \cong \operatorname{Pic}\left(\mathcal{T}_{\bar{k}}^{c}\right)$. En particulier $\operatorname{Pic}\left(\mathcal{T}_{\bar{k}}^{c}\right)$ est un module de permutation de type fini.

(iv) [HS, Thm. 1.6] L'application naturelle $\operatorname{Br}\left(X_{\bar{k}}\right) \rightarrow \operatorname{Br}\left(\mathcal{T}_{\bar{k}}\right)$ est un isomorphisme.

(v) [CTS87, Rem. 2.8.4] Pour toute extension $K / k$ de corps, si $X_{K}$ est projective et $K$-rationnelle, alors $\mathcal{T}_{K}$ est stablement $K$-rationnelle. Si de plus $K=\bar{k}$, alors $\mathcal{T}_{K}$ est rationnelle.

Par la suite spectrale de Hochschild-Serre, on a : $k^{\times} \cong k[\mathcal{T}]^{\times}, \operatorname{Pic}(\mathcal{T})=0$ et $\operatorname{Br}(k) \cong \operatorname{Br}_{1}(\mathcal{T})$, où $\operatorname{Br}_{1}(\mathcal{T}):=\operatorname{Ker}\left(\operatorname{Br}(\mathcal{T}) \rightarrow \operatorname{Br}\left(\mathcal{T}_{\bar{k}}\right)\right)$. De plus, si $X_{\bar{k}}$ est $\bar{k}$-rationnelle, par le Corollaire 2.9 ci-dessous, on a $\operatorname{Br}(k) \cong \operatorname{Br}(\mathcal{T})$.

Corollaire 2.9. Sous les hypothèses du Théorème 2.8, supposons que $k$ est algébriquement clos et $X$ est projective et rationnelle. Alors $\mathcal{T}$ est rationnelle, $\operatorname{Br}(\mathcal{T})=0, H^{1}(\mathcal{T}, \mathbb{Z} / n)=0, H^{2}(\mathcal{T}, \mathbb{Z} / n)=0$ et le groupe $H^{0}\left(\mathcal{T}, \mathcal{K}_{2}\right)$ est uniquement divisible.

Démonstration. Dans ce cas, $T \cong \mathbb{G}_{m}^{r}$ pour un certain $r \in \mathbb{Z}_{>0}$. Puisque $H_{\text {Zari }}^{1}\left(-, \mathbb{G}_{m}\right)=H^{1}\left(-, \mathbb{G}_{m}\right)$, il existe un ouvert $U \subset X$ tel que $\mathcal{T} \times{ }_{X} U \cong U \times \mathbb{G}_{m}^{r}$. Donc $\mathcal{T}$ est rationnelle.

Par le Théorème 2.8 (iv), $\operatorname{Br}(\mathcal{T})=0$. Par la suite de Kummer, et le Théorème 2.8 (i) et (ii), ceci implique $H^{1}(\mathcal{T}, \mathbb{Z} / n)=0$ et $H^{2}(\mathcal{T}, \mathbb{Z} / n)=0$. Par le Théorème 2.1 et le triangle $(2.2)$ sur $\mathbb{Z}(2)$, on $\mathrm{a} 0 \cong$ $H^{1}(\mathcal{T}, \mathbb{Z} / n(2)) \rightarrow H^{0}\left(\mathcal{T}, \mathcal{K}_{2}\right)[n]$ et $H^{0}\left(\mathcal{T}, \mathcal{K}_{2}\right) / n \hookrightarrow H^{2}(\mathcal{T}, \mathbb{Z} / n(2)) \cong 0$. Ainsi $H^{0}\left(\mathcal{T}, \mathcal{K}_{2}\right)$ est uniquement divisible.

Comme conséquence, on a $\bar{k}\left[\mathcal{T}^{c}\right]^{\times} / \bar{k}^{\times}=0, \operatorname{Br}\left(\mathcal{T}_{\bar{k}}^{c}\right)=0$, et $H^{1}\left(\mathcal{T}_{\bar{k}}^{c}, \mathbb{Z} / n\right)=0$, mais ceci résulte déjà de la rationalité de la $\bar{k}$-variété projective et lisse $\mathcal{T}_{\bar{k}}^{c}$.

Corollaire 2.10. Sous les hypothèses du Théorème 2.8, supposons qu'il existe une extension finie $K / k$ de corps de degré d telle que la variété $X_{K}$ soit projective et $K$-rationnelle. Alors, pour tous $i \in \mathbb{N}, j \in \mathbb{Z}$, le groupe $\frac{H_{\mathrm{nr}}^{i}\left(\mathcal{T}^{c}, \mathbb{Q} / \mathbb{Z}(j)\right)}{H^{i}(k, \mathbb{Q} / \mathbb{Z}(j))}$ est annulé pard.

Démonstration. D'après le Théorème $2.8(\mathrm{v})$, la $K$-variété $\mathcal{T}_{K}$ est stablement $K$-rationnelle. Ainsi $\frac{H_{\mathrm{nr}}^{i}\left(\mathcal{T}_{K}^{c}, \mathbb{Q} / \mathbb{Z}(j)\right)}{H^{i}(K, \mathbb{Q} / \mathbb{Z}(j))}$ $=0$ pour tous $i, j$. Puisque le transfert est bien défini pour $H_{\mathrm{nr}}^{i}(-, \mathbb{Q} / \mathbb{Z}(2))$ et $H^{i}(-, \mathbb{Q} / \mathbb{Z}(2))$, on peut définir le transfert $\frac{H_{\mathrm{nr}}^{i}\left(\mathcal{T}_{K}^{c}, \mathbb{Q} / \mathbb{Z}(j)\right)}{H^{i}(K, \mathbb{Q} / \mathbb{Z}(j))} \stackrel{\operatorname{tr}}{\rightarrow} \frac{H_{\mathrm{nr}}^{i}\left(\mathcal{T}^{c}, \mathbb{Q} / \mathbb{Z}(j)\right)}{H^{i}(k, \mathbb{Q} / \mathbb{Z}(j))}$. Un argument de restriction-inflation donne le résultat.

En utilisant la Proposition 2.5(i), dans un précédent article j’ai établi :

Théorème 2.11. ([Cao, Thm. 2.7]) Soit $X$ une $k$-surface projective, lisse, géométriquement rationnelle. Soit $\mathcal{T} \rightarrow$ $X$ un torseur universel sur $X$ et soit $\mathcal{T}^{c}$ une $k$-compactification lisse de $\mathcal{T}$. Alors le groupe $\frac{H_{\mathrm{nr}}^{3}\left(\mathcal{T}^{c}, \mathbb{Q} / \mathbb{Z}(2)\right)}{H^{3}(k, \mathbb{Q} / \mathbb{Z}(2))}$ est fini. 


\section{E. Accouplements avec les 0-cycles}

Soit $X$ une variété projective, lisse, géométriquement intègre. Pour toute extension de corps $K / k$ et tous $i \in \mathbb{N}, j \in \mathbb{Z}$, on a un accouplement naturel :

$$
A_{0}\left(X_{K}\right) \times H_{\mathrm{nr}}^{i}(X, \mathbb{Q} / \mathbb{Z}(j)) \rightarrow H^{i}(K, \mathbb{Q} / \mathbb{Z}(j)):\left(\sum_{i} n_{i} P_{i}, \alpha\right) \mapsto \sum_{i} \operatorname{Norm}_{K\left(P_{i}\right) / K} P_{i}^{*}(\alpha),
$$

où $P_{i}:$ Spec $K\left(P_{i}\right) \rightarrow X$ (voir $[\mathrm{Me} 08$, formule (3)]).

Proposition 2.12. Soit $X$ une $k$-surface projective, lisse, géométriquement rationnelle. Soit $\mathcal{T} \rightarrow X$ un torseur universel sur $X$ et soit $\mathcal{T}^{c}$ une $k$-compactification lisse de $\mathcal{T}$. Supposons que $\mathcal{T}^{c}(k) \neq \emptyset$. Alors l'homomorphisme

$$
H_{\mathrm{nr}}^{i}(X, \mathbb{Q} / \mathbb{Z}(j)) \rightarrow \frac{H_{\mathrm{nr}}^{i}\left(\mathcal{T}^{c}, \mathbb{Q} / \mathbb{Z}(j)\right)}{H^{i}(k, \mathbb{Q} / \mathbb{Z}(j))}
$$

est nul pour tous $i \in \mathbb{N}, j \in \mathbb{Z}$.

Démonstration. Soit $T$ un tore tel que $T^{*} \cong \operatorname{Pic}\left(X_{\bar{k}}\right)$. Alors $\mathcal{T} \rightarrow X$ est un $T$-torseur, i.e. $[\mathcal{T}] \in H^{1}(X, T)$.

Il existe une $T$-variété torique lisse $T^{c}$ (cf. [CTHS, Cor. 1]). Puisque le groupe $H_{\mathrm{nr}}^{i}(\bullet, \mathbb{Q} / \mathbb{Z}(j))$ est un invariant birationnel des variétés projectives et lisses, et que l'existence d'un $k$-point est un invariant $k$ birationnel des $k$-variétés projectives et lisses (lemme de Nishimura), on peut supposer que $\mathcal{T}^{c} \cong T^{c} \times^{T} \mathcal{T}$. Il existe alors un morphisme $\mathcal{T}^{c} \stackrel{\pi}{\rightarrow} X$ étendant $\mathcal{T} \rightarrow X$.

Par [CTS80, §II.B], pour toute extension $K / k$ de corps, $[\mathcal{T}$ ] induit un homomorphisme

$$
A_{0}\left(X_{K}\right) \stackrel{\theta}{\rightarrow} H^{1}(K, T): \sum_{i} x_{i} \mapsto \sum_{i} \operatorname{Res}_{K\left(x_{i}\right) / K} x_{i}^{*}[\mathcal{T}]
$$

Puisque $[\mathcal{T}]_{\mathcal{T}}=0 \in H^{1}(\mathcal{T}, T)$ et que tout élément de $A_{0}\left(\mathcal{T}_{K}^{c}\right)$ équivaut à un élément supporté sur $\mathcal{T}_{K}$ (lemme de déplacement facile sur les zéro-cycles sur une variété lisse), la composition des homomorphismes

$$
A_{0}\left(\mathcal{T}_{K}^{c}\right) \stackrel{\pi_{*}}{\rightarrow} A_{0}\left(X_{K}\right) \stackrel{\theta}{\rightarrow} H^{1}(K, T): \sum_{i} t_{i} \mapsto \sum_{i} \operatorname{Res}_{K\left(t_{i}\right) / K} t_{i}^{*}\left(\pi^{*}[\mathcal{T}]\right)
$$

est nulle.

D'après [CT83, Prop. 4] et [CTS81, Thm. 3], pour toute extension $K / k$ de corps, le morphisme $A_{0}\left(X_{K}\right) \stackrel{\theta}{\rightarrow}$ $H^{1}(K, T)$ est injectif. Ainsi le morphisme $A_{0}\left(\mathcal{T}_{K}^{c}\right) \stackrel{\pi_{*}}{\rightarrow} A_{0}\left(X_{K}\right)$ est nul.

Pour toute extension $K / k$ de corps, on a des accouplements compatibles :

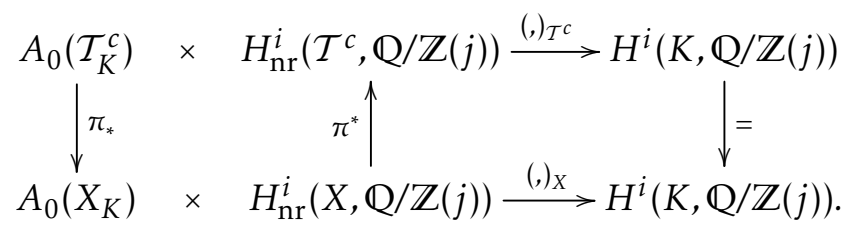

Soit $t \in \mathcal{T}^{c}(k), K:=k(\mathcal{T})$ et $\eta \in \mathcal{T}$ le point générique. Alors $t-\eta \in A_{0}\left(\mathcal{T}_{K}^{c}\right)$ et, pour tout $\alpha \in H_{\mathrm{nr}}^{i}(X, \mathbb{Q} / \mathbb{Z}(j))$, on a

$$
\left(t, \pi^{*} \alpha\right)_{\mathcal{T}^{c}}-\left(\eta, \pi^{*} \alpha\right)_{\mathcal{T}^{c}}=\left(t-\eta, \pi^{*} \alpha\right)_{\mathcal{T}^{c}}=\left(\pi_{*}(t-\eta), \alpha\right)_{X}=(0, \alpha)_{X}=0 \in H^{i}(K, \mathbb{Q} / \mathbb{Z}(j)) .
$$

Puisque $(\eta,-)_{\mathcal{T}}$ est l'inclusion canonique $H_{\mathrm{nr}}^{i}\left(\mathcal{T}^{c}, \mathbb{Q} / \mathbb{Z}(j)\right) \subset H^{i}\left(k\left(\mathcal{T}^{c}\right), \mathbb{Q} / \mathbb{Z}(j)\right)$ et

$$
\operatorname{Im}(t,-)_{\mathcal{T}^{c}} \subset \operatorname{Im}\left(H^{i}(k, \mathbb{Q} / \mathbb{Z}(j)) \rightarrow H^{i}(K, \mathbb{Q} / \mathbb{Z}(j))\right),
$$

on a $\operatorname{Im}\left(\pi^{*}\right) \in \operatorname{Im}\left(H^{i}(k, \mathbb{Q} / \mathbb{Z}(j)) \rightarrow H_{\mathrm{nr}}^{i}\left(\mathcal{T}^{c}, \mathbb{Q} / \mathbb{Z}(j)\right)\right)$. 


\section{Cohomologie motivique à coefficients $\mathbb{Z}(2)$ d'un torseur sous un tore}

Dans [Me16], A. Merkurjev a étudié la cohomologie motivique à coefficients $\mathbb{Z}(2)$ pour un torseur sous un groupe semisimple. Nous reprenons sa méthode pour étudier les torseurs sous un tore. Soient $T$ un tore sur $k$ de dimension $N$, et $f: Y \rightarrow X$ un torseur sous $T$ sur $X$, où $X$ est une $k$-variété lisse géométriquement intègre. On calcule la relation entre la cohomologie motivique de $X$ et celle de $Y$ à coefficients dans le complexe $\mathbb{Z}(2)$, i.e. on calcule la cohomologie de $\mathbb{Z}_{f}(2)$ ci-dessous (Théorème 3.6).

La plan de la démonstraction du Théorème 3.6 est : (i) on calcule les faisceaux cohomologiques de $\mathbb{Z}_{f}(2)$, en utilisant une méthode de Merkurjev ([Me16]), qui généralise le travail de Sansuc sur $\mathbb{Z}_{f}(1)$; (ii) on calcule la cohomologie de $\mathbb{Z}_{f}(2)$ sur $X_{\bar{k}}$. Ce théorème sera appliqué à calculer la cohomologie de $\mathbb{Z}_{f}(2)$ sur $X$ (Théorème 4.7) en utilisant la suite spectrale de Hochschild-Serre.

Dans ce cas, on définit $\mathbb{Z}_{f}(i)$ le cône du morphisme naturel $\mathbb{Z}_{X}(i) \rightarrow R f_{*} \mathbb{Z}_{Y}(i)$ pour $i=1,2$ ([Me16, (4.3)]), i.e. on a deux triangles dans $D_{e t}^{+}(X)$ :

$$
\mathbb{Z}_{X}(1) \rightarrow R f_{*} \mathbb{Z}_{Y}(1) \rightarrow \mathbb{Z}_{f}(1) \rightarrow \mathbb{Z}_{X}(1)[1]
$$

et

$$
\mathbb{Z}_{X}(2) \stackrel{f^{\sharp}(2)}{\longrightarrow} R f_{*} \mathbb{Z}_{Y}(2) \stackrel{d(2)}{\longrightarrow} \mathbb{Z}_{f}(2) \rightarrow \mathbb{Z}_{X}(2)[1] .
$$

Pour chaque $\Gamma_{k}$-module $M$ continu discret, on peut voir $M$ comme un faisceau étale sur le petit site étale (ét $/ k$ ), et son image inverse sur le grand site étale est noté également par $M$. Ainsi $\wedge^{*} T^{*}$ est un faisceau étale.

Soient

$$
R^{\leq 2} f_{*} \mathbb{Z}_{Y}(1):=\tau^{\leq 2} R f_{*} \mathbb{Z}_{Y}(1) \quad \text { et } \quad \mathbb{Z}_{f}^{\leq 2}(1):=\tau^{\leq 2} \mathbb{Z}_{f}(1) .
$$

Proposition 3.1. On a $\mathbb{Z}_{f}^{\leq 2}(1) \cong T^{*}[-1]$ et donc $\mathbb{Z}_{X}(1) \otimes \mathbb{Z}_{f}^{\leq 2}(1) \cong \mathbb{G}_{m} \otimes T^{*}[-2]$ et

$$
\tau^{\leq 2}\left(\mathbb{Z}_{X}(1) \otimes \mathbb{Z}_{f}(1)\right) \cong \mathbb{G}_{m} \otimes T^{*}[-2]
$$

Démonstration. Puisque $\mathbb{Z}_{X}(1)=\left.\mathbb{G}_{m}[-1]\right|_{X}$ et $\mathbb{Z}_{Y}(1)=\left.\mathbb{G}_{m}[-1]\right|_{Y}$, on a : $\left.\mathcal{H}^{1}\left(\mathbb{Z}_{X}(1)\right) \cong \mathbb{G}_{m}\right|_{X}, \mathcal{H}^{i}\left(\mathbb{Z}_{X}(1)\right)=$ 0 pour tout $i \neq 1$ et $\mathcal{H}^{i}\left(R f_{*} \mathbb{Z}_{Y}(1)\right)=R^{i-1} f_{*}\left(\left.\mathbb{G}_{m}\right|_{Y}\right)$ pour tout $i$. Ainsi $\mathcal{H}^{i}\left(R f_{*} \mathbb{Z}_{Y}(1)\right)=0$ pour $i \leq 0$ et le faisceau $\mathcal{H}^{2}\left(R f_{*} \mathbb{Z}_{Y}(1)\right)$ est le faisceau étale associé au préfaisceau :

$$
U \longmapsto \mathbb{H}^{2}\left(f^{-1} U, \mathbb{Z}(1)\right)=\operatorname{Pic}\left(f^{-1} U\right)
$$

Localement pour la topologie étale, $f^{-1} U \stackrel{\sim}{\rightarrow} U \times \mathbb{G}_{m}^{N}$ et $U$ est le spectre d'un anneau local régulier. Dans ce cas on a $0 \cong \operatorname{Pic}(U) \cong \operatorname{Pic}\left(f^{-1} U\right)$. Donc $\mathcal{H}^{2}\left(R f_{*} \mathbb{Z}_{Y}(1)\right)=0$. Ainsi

$$
\mathcal{H}^{i}\left(\mathbb{Z}_{X}(1)\right)=0 \text { pour } i \neq 1, \quad \mathcal{H}^{i}\left(R f_{*} \mathbb{Z}_{Y}(1)\right)=0 \text { pour } i \leq 0 \text { et } i=2 .
$$

D’après (3.1), on a une suite exacte longue :

$$
\cdots \rightarrow \mathcal{H}^{i}\left(\mathbb{Z}_{X}(1)\right) \stackrel{\phi_{i}}{\longrightarrow} \mathcal{H}^{i}\left(R f_{*} \mathbb{Z}_{Y}(1)\right) \rightarrow \mathcal{H}^{i}\left(\mathbb{Z}_{f}(1)\right) \rightarrow \mathcal{H}^{i+1}\left(\mathbb{Z}_{X}(1)\right) \stackrel{\phi_{i+1}}{\longrightarrow} \cdots
$$

D'après [CTS87, Prop. 1.4.2], on a une suite exacte :

$$
0 \rightarrow \mathbb{G}_{m, X} \rightarrow f_{*} \mathbb{G}_{m, Y} \rightarrow T^{*} \rightarrow 0
$$

et donc $\phi_{1}$ est injectif de conoyau $T^{*}$. D'après (3.4), on a $\mathcal{H}^{1}\left(\mathbb{Z}_{f}(1)\right) \cong T^{*}$ et $\mathcal{H}^{i}\left(\mathbb{Z}_{f}(1)\right)=0$ pour $i \leq 0$ et $i=2$. Ceci donne le premier énoncé.

Le deuxième énoncé résulte du fait que : $T^{*} \otimes L-\cong T^{*} \otimes-$.

Le troisième énoncé résulte du fait que : d'après (1.1), on a $\mathbb{G}_{m} \otimes^{L}\left(D_{e ̂ t}^{\geq 3}(X)\right) \subset D_{e ̂ t}^{\geq 2}(X)$. 
La Proposition 3.1 induit un triangle dans $D_{e ̂ t}^{+}(X)$ :

$$
\mathbb{Z}_{X}(1) \stackrel{f^{\sharp}(1)}{\longrightarrow} R^{\leq 2} f_{*} \mathbb{Z}_{Y}(1) \stackrel{d(1)}{\longrightarrow} \mathbb{Z}_{f}^{\leq 2}(1) \rightarrow \mathbb{Z}_{X}(1)[1]
$$

et un isomorphisme :

$$
\mathbb{G}_{m} \otimes T^{*} \cong \mathcal{H}^{2}\left(\mathbb{Z}_{X}(1) \otimes \mathbb{Z}_{f}^{\leq 2}(1)\right) \cong \mathcal{H}^{2}\left(\mathbb{Z}_{X}(1) \otimes^{L} \mathbb{Z}_{f}(1)\right)
$$

Si $Y \cong X \times T$, la projection $X \times T \rightarrow T$ induit une section de (3.6) :

$$
T^{*}[-1] \cong \tau^{\leq 2} \mathbb{Z}_{f}(1) \rightarrow R f_{*} \mathbb{Z}_{Y}(1) .
$$

Le résultat principal de cette section est la Proposition 3.5, qui calcule $\mathbb{Z}_{f}(2)$.

Soit $T_{0}:=\mathbb{G}_{m}^{N}$. Puisque $H^{0}\left(T_{0}, \mathcal{K}_{1}\right)=k\left[T_{0}\right]^{\times} \supset T_{0}^{*}$, ceci induit un homomorphisme canonique $T_{0}^{*} \rightarrow$ $H^{0}\left(T_{0}, \mathcal{K}_{1}\right)$. Pour chaque variété lisse intègre $U$ et tout $n \in \mathbb{N}$, le cup-produit

$$
\left[H^{n}\left(U, \mathcal{K}_{2}\right) \otimes H^{0}\left(T_{0}, \mathcal{K}_{0}\right)\right] \oplus\left[H^{n}\left(U, \mathcal{K}_{1}\right) \otimes H^{0}\left(T_{0}, \mathcal{K}_{1}\right)\right] \stackrel{\cup}{\rightarrow} H^{n}\left(U \times T_{0}, \mathcal{K}_{2}\right)
$$

induit un homomorphisme :

$$
H^{n}\left(U, \mathcal{K}_{2}\right) \oplus\left[H^{n}\left(U, \mathcal{K}_{1}\right) \otimes T_{0}^{*}\right] \stackrel{\cup}{\rightarrow} H^{n}\left(U \times T_{0}, \mathcal{K}_{2}\right) .
$$

Lemme 3.2. Soit $T_{0}:=\mathbb{G}_{m}^{N}$ et $U$ une variété lisse intègre. Alors, pour tout $n \in \mathbb{N}$, on a une suite exacte canonique (où $\cup$ est défini dans (3.9)) :

$$
0 \rightarrow H^{0}\left(U, \mathcal{K}_{2}\right) \oplus\left[H^{0}\left(U, \mathcal{K}_{1}\right) \otimes T_{0}^{*}\right] \stackrel{\cup}{\rightarrow} H^{0}\left(U \times T_{0}, \mathcal{K}_{2}\right) \rightarrow H^{0}\left(U, \mathcal{K}_{0}\right) \otimes \wedge^{2} T_{0}^{*} \rightarrow 0 .
$$

et un isomorphisme $H^{n}\left(U, \mathcal{K}_{2}\right) \oplus\left[H^{n}\left(U, \mathcal{K}_{1}\right) \otimes T_{0}^{*}\right] \stackrel{\cup}{\rightarrow} H^{n}\left(U \times T_{0}, \mathcal{K}_{2}\right)$ pour $n \geq 1$.

Démonstration. D'après $(2.1)$, on a $H^{n}\left(U, \mathcal{K}_{0}\right)=0$ pour $n \geq 1$.

Notons $K_{i}^{M}$ les groupes de $K$-théorie algébrique de Milnor. Par [Me03, §4], pour toute variété lisse $X$, on a un complexe de Gersten :

$$
0 \rightarrow \oplus_{x \in X^{(0)}} K_{j}^{M}(k(x)) \rightarrow \oplus_{x \in X^{(1)}} K_{j-1}^{M}(k(x)) \rightarrow \cdots \rightarrow \oplus_{x \in X^{(j)}} \mathbb{Z} \rightarrow 0
$$

et on note $A^{i}\left(X, K_{j}^{M}\right)$ les groupes de cohomologie de ce complexe. Donc $A^{i}\left(X, K_{0}^{M}\right)=0$ pour $i \geq 1$.

Soit $f_{1}, \ldots, f_{N}$ une base de $T_{0}^{*}$. Ainsi $\left\{f_{i_{1}}, \ldots, f_{i_{q}}\right\}_{1 \leq i_{1}<\cdots<i_{q} \leq N}$ est un élément de $A^{0}\left(T_{0}, K_{q}\right)$. Le $A^{*}\left(U, K_{*}\right)$ module $A^{*}\left(U \times T_{0}, K_{*}\right)$ est libre avec une base consistant en les éléments $\left\{f_{i_{1}}, \ldots, f_{i_{q}}\right\}$ pour $q=0,1, \cdots, N$ et tout $q$-uplet $1 \leq i_{1}<i_{2}<\cdots<i_{q} \leq N$. Pour $N=1$, ceci est [Me03, Proposition 5.5]. Le cas général en découle par récurrence (cf. [Me03, Corollaire 5.6]).

Pour $K_{2}^{M}$, on a

$$
A^{n}\left(U \times T_{0}, K_{2}^{M}\right) \cong A^{n}\left(U, K_{2}^{M}\right) \oplus\left[\oplus_{i} A^{n}\left(U, K_{1}^{M}\right) \cup\left\{f_{i}\right\}\right] \oplus\left[\oplus_{i<j} A^{n}\left(U, K_{0}^{M}\right) \cup\left\{f_{i}, f_{j}\right\}\right] .
$$

Alors le cup-produit induit une injection (l'analogue de (3.9))

$$
A^{n}\left(U, K_{2}^{M}\right) \oplus\left[A^{n}\left(U, K_{1}^{M}\right) \otimes T_{0}^{*}\right] \stackrel{\cup_{1,1}}{\hookrightarrow} A^{n}\left(U \times T_{0}, K_{2}^{M}\right)
$$

et $\operatorname{coker}\left(\cup_{1,1}\right) \cong \oplus_{i<j} A^{n}\left(U, K_{0}^{M}\right) \cup\left\{f_{i}, f_{j}\right\}$. Donc le morphisme (cf. [Me03, (5.8)]) :

$$
A^{n}\left(U, K_{0}^{M}\right) \otimes \wedge^{2} T_{0}^{*} \rightarrow \operatorname{coker}\left(\cup_{1,1}\right): a \otimes\left(f_{i} \wedge f_{j}\right) \rightarrow a \cup\left\{f_{i}, f_{j}\right\}
$$


est bien défini et c'est un isomorphisme. Ceci donne une suite exacte

$$
0 \rightarrow A^{n}\left(U, K_{2}^{M}\right) \oplus\left[A^{n}\left(U, K_{1}^{M}\right) \otimes T_{0}^{*}\right] \stackrel{\cup}{\rightarrow} A^{n}\left(U \times T_{0}, K_{2}^{M}\right) \rightarrow A^{n}\left(U, K_{0}^{M}\right) \otimes \wedge^{2} T_{0}^{*} \rightarrow 0 .
$$

Donc $\cup: A^{n}\left(U, K_{2}^{M}\right) \oplus\left[A^{n}\left(U, K_{1}^{M}\right) \otimes T_{0}^{*}\right] \stackrel{\sim}{\rightarrow} A^{n}\left(U \times T_{0}, K_{2}^{M}\right)$ pour $n \geq 1$.

Pour tout corps $F$ et $i=0,1,2$, on a $K_{i}^{M}(F) \stackrel{\sim}{\rightarrow} K_{i}(F)$ (voir D3 dans [Ro96, (1.12)]). D'après (2.1), pour $j=0,1,2$, on a donc un isomorphisme canonique $A^{i}\left(X, K_{j}^{M}\right) \stackrel{\sim}{\rightarrow} H^{i}\left(X, \mathcal{K}_{j}\right)$. Ceci donne le résultat annoncé.

Lemme 3.3. Les faisceaux $\mathcal{H}^{i}\left(\mathbb{Z}_{f}(2)\right)$ sont nuls pour $i \leq 1$ et $i=3$.

Démonstration. On suit la démonstration dans [Me16, pp. 10]

Puisque $\mathbb{Z}_{X}(2)$ et $\mathbb{Z}_{Y}(2)$ sont supportés en degrés 1 et 2 , on a $\mathcal{H}^{i}\left(\mathbb{Z}_{f}(2)\right)=0$ pour $i<0, R^{i} f_{*} \mathbb{Z}_{Y}(2)=0$ pour $i \leq 0, R^{i} f_{*} \mathbb{Z}_{Y}(2) \cong \mathcal{H}^{i}\left(\mathbb{Z}_{f}(2)\right)$ pour $i \geq 3$ et $\mathcal{H}^{3}\left(\mathbb{Z}_{X}(2)\right)=0$. Ainsi on a une suite exacte longue dans la catégorie des faisceaux étales sur $X$ :

$$
\begin{gathered}
0 \longrightarrow \mathcal{H}^{0}\left(\mathbb{Z}_{f}(2)\right) \longrightarrow \mathcal{H}^{1}\left(\mathbb{Z}_{X}(2)\right) \stackrel{s}{\longrightarrow} R^{1} f_{*} \mathbb{Z}_{Y}(2) \longrightarrow \\
\mathcal{H}^{1}\left(\mathbb{Z}_{f}(2)\right) \longrightarrow \mathcal{H}^{2}\left(\mathbb{Z}_{X}(2)\right) \stackrel{s_{1}}{\longrightarrow} R^{2} f_{*} \mathbb{Z}_{Y}(2) \longrightarrow \mathcal{H}^{2}\left(\mathbb{Z}_{f}(2)\right) \longrightarrow 0
\end{gathered}
$$

Les faisceaux $\mathcal{H}^{1}\left(\mathbb{Z}_{X}(2)\right)$ et $R^{1} f_{*} \mathbb{Z}_{Y}(2)$ sont les faisceaux étales associés aux préfaisceaux :

$$
U \longmapsto \mathbb{H}^{1}(U, \mathbb{Z}(2))=K_{3, \text { ind }} k(U) \text { et } U \longmapsto \mathbb{H}^{1}\left(f^{-1} U, \mathbb{Z}(2)\right)=K_{3, \text { ind }} k\left(f^{-1} U\right) .
$$

Localement pour la topologie étale, $f^{-1} U \stackrel{\sim}{\rightarrow} U \times \mathbb{G}_{m}^{N}$, et dans ce cas on a $K_{3, \text { ind }} k(U) \cong K_{3, \text { ind }} k\left(f^{-1} U\right)$, car $K_{3, \text { ind }} k(U) \cong K_{3, \text { ind }} k\left(U \times \mathbb{G}_{m}^{N}\right)$ (cf. [EKLV, Lem. 6.2]). Donc $s$ est un isomorphisme.

Les faisceaux $\mathcal{H}^{2}\left(\mathbb{Z}_{X}(2)\right)$ et $R^{2} f_{*} \mathbb{Z}_{Y}(2)$ sont les faisceaux étales associés aux préfaisceaux :

$$
U \longmapsto \mathbb{H}^{2}(U, \mathbb{Z}(2))=H^{0}\left(U, \mathcal{K}_{2}\right) \text { et } U \longmapsto \mathbb{H}^{2}\left(f^{-1} U, \mathbb{Z}(2)\right)=H^{0}\left(f^{-1} U, \mathcal{K}_{2}\right) .
$$

Localement pour la topologie étale, $f^{-1} U \stackrel{\sim}{\rightarrow} U \times \mathbb{G}_{m}^{N}$, et dans ce cas le morphisme canonique $H^{0}\left(U, \mathcal{K}_{2}\right) \rightarrow$ $H^{0}\left(f^{-1} U, \mathcal{K}_{2}\right)$ est injectif puisque que $f^{-1} U \rightarrow U$ admet alors une section. Donc $s_{1}$ est injectif.

On a donc établi $\mathcal{H}^{i}\left(\mathbb{Z}_{f}(2)\right)=0$ pour $i \leq 1$.

Le faisceau $R^{3} f_{*} \mathbb{Z}_{Y}(2)$ est le faisceau étale associé au préfaisceau :

$$
U \longmapsto \mathbb{H}^{3}\left(f^{-1} U, \mathbb{Z}(2)\right)=H^{1}\left(f^{-1} U, \mathcal{K}_{2}\right) .
$$

Localement pour la topologie étale, $f^{-1} U \stackrel{\sim}{\rightarrow} U \times \mathbb{G}_{m}^{N}$. Notons $T_{0}:=\mathbb{G}_{m}^{N}$. Dans ce cas, par le lemme 3.2 , on a un isomorphisme :

$$
H^{1}\left(U, \mathcal{K}_{2}\right) \oplus\left[H^{1}\left(U, \mathcal{K}_{1}\right) \otimes T_{0}^{*}\right] \rightarrow H^{1}\left(f^{-1} U, \mathcal{K}_{2}\right)
$$

Puisque $\mathcal{K}_{*}$ est un faisceau de Zariski, le faisceau associé à $\left\{U \longmapsto H^{1}\left(U, \mathcal{K}_{*}\right)\right\}$ est 0 et donc le faisceau associé à $\left\{U \longmapsto H^{1}\left(f^{-1} U, \mathcal{K}_{2}\right)\right\}$ est 0 . Ainsi $R^{3} f_{*} \mathbb{Z}_{Y}(2)$ est 0 , et $\mathcal{H}^{3}\left(\mathbb{Z}_{f}(2)\right)=0$.

Notons $\mathbb{Z}_{f}(\otimes)$ le cône du morphisme $\mathbb{Z}_{X}(1) \otimes^{L} \mathbb{Z}_{X}(1) \rightarrow R^{\leq 2} f_{*} \mathbb{Z}_{Y}(1) \otimes^{L} R^{\leq 2} f_{*} \mathbb{Z}_{Y}(1)$. Soit $\theta$ la composition des morphismes

$$
\begin{gathered}
R^{\leq 2} f_{*} \mathbb{Z}_{Y}(1) \otimes^{L} R^{\leq 2} f_{*} \mathbb{Z}_{Y}(1) \rightarrow R f_{*} \mathbb{Z}_{Y}(1) \otimes^{L} R f_{*} \mathbb{Z}_{Y}(1) \stackrel{\theta_{1}}{\longrightarrow} \\
R f_{*}\left(\mathbb{Z}_{Y}(1) \otimes^{L} f^{*} R f_{*} \mathbb{Z}_{Y}(1)\right) \stackrel{\theta_{2}}{\longrightarrow} R f_{*}\left(\mathbb{Z}_{Y}(1) \otimes^{L} \mathbb{Z}_{Y}(1)\right) \rightarrow R f_{*} \mathbb{Z}_{Y}(2) .
\end{gathered}
$$


où $\theta_{1}$ est le morphisme canonique induit par $\otimes^{L}$ (cf. [F, p. 306]) et $\theta_{2}$ est induit par le morphisme d'adjonction $f^{*} R f_{*} \mathbb{Z}_{Y}(1) \rightarrow \mathbb{Z}_{Y}(1)$. Le morphisme $\theta$ induit un diagramme commutatif de triangles :

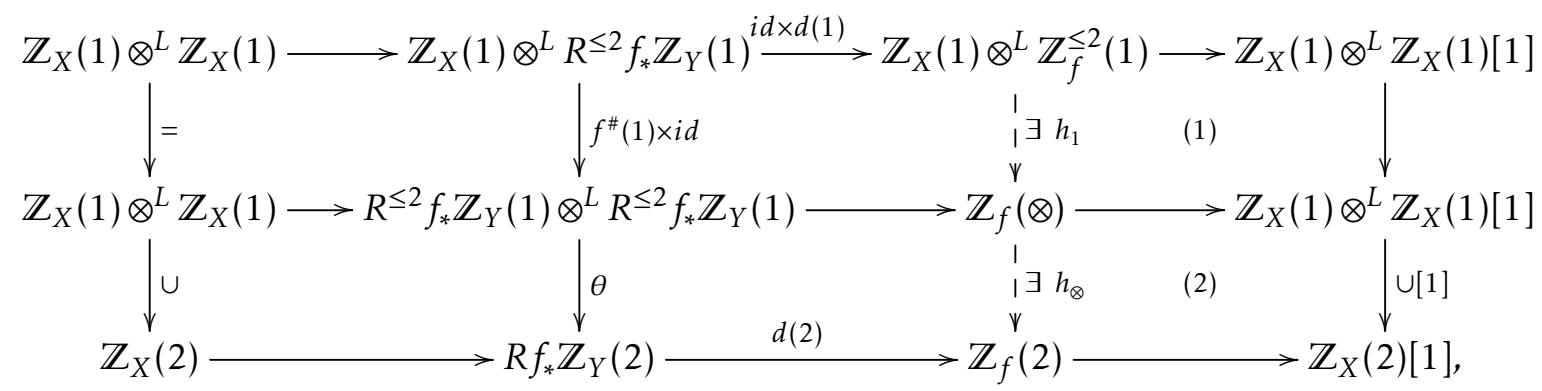

où $h_{1}$ et $h_{\otimes}$ sont donnés par les axiomes des catégories triangulées. Le morphisme $h_{\otimes} \circ h_{1}$ induit un morphisme

$$
h_{1,1}: \mathbb{G}_{m, X} \otimes T^{*} \stackrel{(3.7)}{=} \mathcal{H}^{2}\left(\mathbb{Z}_{X}(1) \otimes^{L} \mathbb{Z}_{f}^{\leq 2}(1)\right) \rightarrow \mathcal{H}^{2}\left(\mathbb{Z}_{f}(2)\right)
$$

Par le Lemme 3.2, il y a une composition de morphismes de groupes

$$
H^{0}\left(Y \times T, \mathcal{K}_{2}\right) \rightarrow H^{0}\left((Y \times T)_{\bar{k}}, \mathcal{K}_{2}\right)^{\Gamma_{k}} \rightarrow\left(H^{0}\left(Y_{\bar{k}}, \mathcal{K}_{0}\right) \otimes \wedge^{2} T^{*}\right)^{\Gamma_{k}} \cong\left(\wedge^{2} T^{*}\right)^{\Gamma_{k}} \stackrel{\sim}{\rightarrow}\left(\wedge^{2} T^{*}\right)(X),
$$

où $\left(\wedge^{2} T^{*}\right)(X)$ est le groupe de sections du faisceau $\left(\wedge^{2} T^{*}\right)$ sur $X$. Définissons :

$$
h_{0,2}^{\prime}(X): H^{0}\left(Y, \mathcal{K}_{2}\right) \stackrel{\rho^{*}}{\rightarrow} H^{0}\left(Y \times T, \mathcal{K}_{2}\right) \rightarrow\left(\wedge^{2} T^{*}\right)(Y) \cong\left(\wedge^{2} T^{*}\right)(X),
$$

où $\rho: Y \times T \rightarrow Y:(y, t) \mapsto t \cdot y$ est l'action. Ceci induit un morphisme de faisceaux :

$$
h_{0,2}^{\prime}: R^{2} f_{*} \mathbb{Z}_{Y}(2) \rightarrow \wedge^{2} T^{*}
$$

Lemme 3.4. Supposons que $T \cong \mathbb{G}_{m}^{N}$ et $Y \rightarrow X$ est un $T$-torseur trivial. Alors le complexe

$$
0 \rightarrow \mathcal{H}^{2}\left(\mathbb{Z}_{X}(2)\right) \oplus\left(\mathbb{G}_{m, X} \otimes T^{*}\right) \stackrel{\left(f^{\sharp}(2), h_{1,1}\right)}{\longrightarrow} R^{2} f_{*} \mathbb{Z}_{Y}(2) \stackrel{h_{0,2}^{\prime}}{\longrightarrow} \wedge^{2} T^{*} \rightarrow 0
$$

est exactement la suite exacte de faisceaux étales associé à la suite exacte de préfaisceaux qui envoie $U \in \mathrm{Sch} / X$ à la suite dans le Lemme 3.2.

Démonstration. Dans le diagramme (3.10), la section (3.8) induit une section de $i d \times d(1)$ :

$$
s: \mathbb{Z}_{X}(1) \otimes \mathbb{Z}_{f}(1) \rightarrow \mathbb{Z}_{X}(1) \otimes{ }^{L} R^{\leq 2} f_{*} \mathbb{Z}_{Y}(1) .
$$

Donc $h_{1,1}$ est la composition $d(2) \circ \theta \circ\left(f^{\#}(1) \times i d\right) \circ s$. D'après (2.4), $\theta$ est induit par le cup-produit de groupes de K-théorie $H^{0}\left(U \times T, \mathcal{K}_{1}\right) \otimes H^{0}\left(U \times T, \mathcal{K}_{1}\right) \rightarrow H^{0}\left(U \times T, \mathcal{K}_{2}\right)$ pour tout $U$ sur $X$, et donc $\left(f^{\#}(2), h_{1,1}\right)$ est le morphisme induit par (3.9) pour tout $U$ sur $X$.

Pour tout $U \in \mathrm{Sch} / X$, soit $\phi_{U}: H^{0}\left(U \times T, \mathcal{K}_{2}\right) \rightarrow \wedge^{2} T^{*}$ le morphisme dans le Lemme 3.2. Ceci induit un diagramme avec le carré (5) commutatif

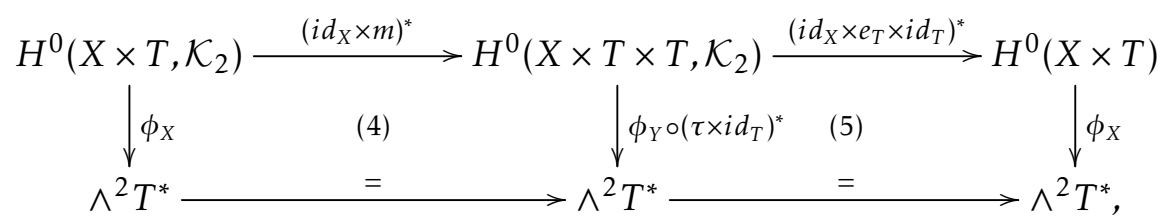

où $m: T \times T \rightarrow T$ la multiplication et $\tau: Y \cong X \times T$ une trivialisation. Puisque $\left(i d_{X} \times m\right) \circ\left(i d_{X} \times e_{T} \times i d_{T}\right)=$ $i d_{X \times T}$, le carré (4) est aussi commutatif. Donc $h_{0,2}^{\prime}(X)=\phi_{X}$, et $h_{0,2}^{\prime}$ est le morphisme associé à $\phi_{U}$ pour tout $U \in \mathrm{Sch} / X$. 
D'après le Lemme 3.4, la composition $\mathcal{H}^{2}\left(\mathbb{Z}_{X}(2)\right) \rightarrow R^{2} f_{*} \mathbb{Z}_{Y}(2) \stackrel{h_{0,2}^{\prime}}{\longrightarrow} \wedge^{2} T^{*}$ est nulle et il existe un morphisme

$$
h_{0,2}: \mathcal{H}^{2}\left(\mathbb{Z}_{f}(2)\right) \rightarrow \wedge^{2} T^{*}
$$

Nous pouvons maintenant établir :

Proposition 3.5. On a $\tau^{\leq 3} \mathbb{Z}_{f}(2) \cong \mathcal{H}^{2}\left(\mathbb{Z}_{f}(2)\right)[-2]$ et une suite exacte de faisceaux étales sur $X$ :

$$
0 \longrightarrow \mathbb{G}_{m, X} \otimes T^{*} \stackrel{h_{1,1}}{\longrightarrow} \mathcal{H}^{2}\left(\mathbb{Z}_{f}(2)\right) \stackrel{h_{0,2}}{\longrightarrow} \wedge^{2} T^{*} \longrightarrow 0 .
$$

Démonstration. D'après le Lemme 3.3, il suffit de montrer que localement pour la topologie étale la suite est exacte. Localement pour la topologie étale, $Y \stackrel{\sim}{\rightarrow} X \times \mathbb{G}_{m}^{N}$, et le résultat découle donc du Lemme 3.4.

Explicitons ce que donne la cohomologie de cette suite exacte de faisceaux.

Théorème 3.6. Soient $X$ une $k$-variété lisse géométriquement connexe, $T$ un $k$-tore et $f: Y \rightarrow X$ un $T$-torseur. Alors on a deux suites exactes longues de $\Gamma_{k}$-modules :

$$
0 \rightarrow \bar{k}[X]^{\times} \otimes T^{*} \rightarrow \mathbb{H}^{2}\left(X_{\bar{k}}, \mathbb{Z}_{f}(2)\right) \rightarrow \wedge^{2} T^{*} \stackrel{h}{\rightarrow} \operatorname{Pic}\left(X_{\bar{k}}\right) \otimes T^{*} \rightarrow \mathbb{H}^{3}\left(X_{\bar{k}}, \mathbb{Z}_{f}(2)\right) \rightarrow 0
$$

$e t$

$$
\begin{aligned}
& 0 \longrightarrow H^{0}\left(X_{\bar{k}}, \mathcal{K}_{2}\right) \longrightarrow H^{0}\left(Y_{\bar{k}}, \mathcal{K}_{2}\right) \longrightarrow \mathbb{H}^{2}\left(X_{\bar{k}}, \mathbb{Z}_{f}(2)\right) \longrightarrow H^{1}\left(X_{\bar{k}}, \mathcal{K}_{2}\right) \\
& \longrightarrow H^{1}\left(Y_{\bar{k}}, \mathcal{K}_{2}\right) \longrightarrow \mathbb{H}^{3}\left(X_{\bar{k}}, \mathbb{Z}_{f}(2)\right) \longrightarrow \mathbb{H}^{4}\left(X_{\bar{k}}, \mathbb{Z}_{X}(2)\right) \longrightarrow \mathbb{H}^{4}\left(Y_{\bar{k}}, \mathbb{Z}_{Y}(2)\right),
\end{aligned}
$$

où :

(1) le morphisme $h$ est donné par : $a \wedge b \mapsto a \otimes \partial(b)-b \otimes \partial(a)$ avec $\partial: T^{*} \rightarrow \operatorname{Pic}\left(X_{\bar{k}}\right)$ le morphisme associé au torseur $Y \rightarrow X$ sous le tore $T$ (induit par (3.5));

(2) la composition de morphismes

$$
\operatorname{Pic}\left(X_{\bar{k}}\right) \otimes T^{*} \rightarrow \mathbb{H}^{3}\left(X_{\bar{k}}, \mathbb{Z}_{f}(2)\right) \rightarrow \mathbb{H}^{4}\left(X_{\bar{k}}, \mathbb{Z}_{X}(2)\right),
$$

est induite par l'intersection :

$$
\operatorname{Pic}\left(X_{\bar{k}}\right) \otimes T^{*} \stackrel{i d \times \partial}{\longrightarrow} \operatorname{Pic}\left(X_{\bar{k}}\right) \times \operatorname{Pic}\left(X_{\bar{k}}\right) \rightarrow \mathrm{CH}^{2}\left(X_{\bar{k}}\right) \rightarrow \mathbb{H}^{4}\left(X_{\bar{k}}, \mathbb{Z}_{X}(2)\right) .
$$

Démonstration. Sur $\bar{k}$, on a $T^{*} \cong \mathbb{Z}^{N}$, donc

$$
H^{1}\left(X_{\bar{k}}, \wedge^{2} T^{*}\right)=0 \quad \text { et } \quad H^{i}\left(X_{\bar{k}}, \mathbb{G}_{m, X} \otimes T^{*}\right) \cong H^{i}\left(X_{\bar{k}}, \mathbb{G}_{m, X}\right) \otimes T^{*}
$$

(le premier énoncé utilisant la lissité de $X)$. D'après la Proposition $3.5, \mathbb{H}^{i}\left(X_{\bar{k}}, \mathbb{Z}_{f}(2)\right) \cong H^{i-2}\left(X_{\bar{k}}, \mathcal{H}^{2}\left(\mathbb{Z}_{f}(2)\right)\right.$ ) pour chaque $i \leq 3$. En appliquant $H^{i}\left(X_{\bar{k}},-\right)$ aux suites exactes (3.2) et (3.11), on déduit les deux suites exactes longues.

La composition dans l'énoncé (2) est induite par $\mathbb{Z}_{X}(1) \otimes^{L} \mathbb{Z}_{f}^{\leq 2}(1) \rightarrow \mathbb{Z}_{f}(2) \rightarrow \mathbb{Z}_{X}(2)[1]$. D'après les carrés (1), (2) dans le diagramme (3.10) et (2.5), on a un diagramme commutatif :

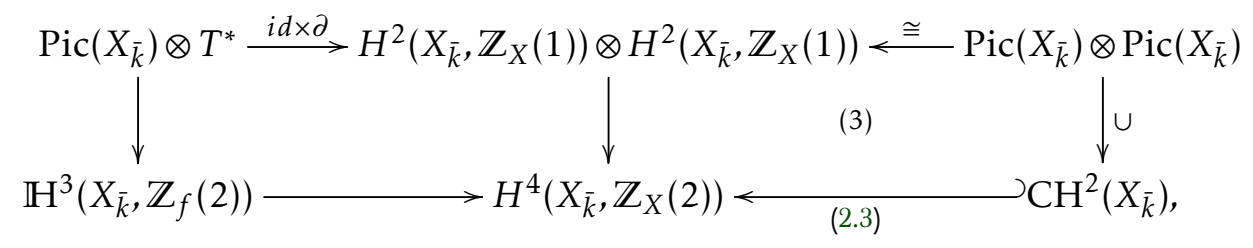


où $\cup$ est l'intersection. L'énoncé (2) en découle.

Établissons l'énoncé (1). On a un diagramme commutatif de triangles :

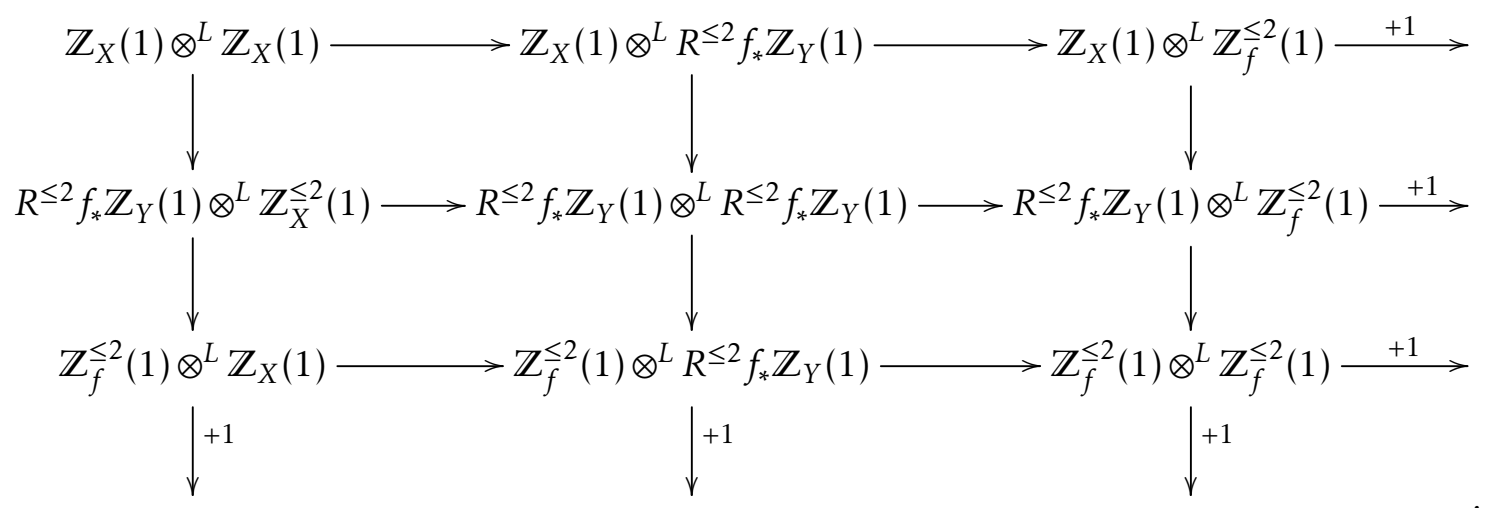

Par la définition de $\mathbb{Z}_{f}(\otimes)$ et la démonstration de [BBD, Prop. 1.1.11] appliqué au diagramme ci-dessus, on a les triangles ([BBD, pp. 25, Diagramme (1) et (2)]) :

$$
\begin{aligned}
& \mathbb{Z}_{X}(1) \otimes^{L} \mathbb{Z}_{f}^{\leq 2}(1) \stackrel{h_{1}}{\longrightarrow} \mathbb{Z}_{f}(\otimes) \rightarrow \mathbb{Z}_{f}^{\leq 2}(1) \otimes^{L} R^{\leq 2} f_{*} \mathbb{Z}_{Y}(1) \stackrel{+1}{\longrightarrow}, \\
& \mathbb{Z}_{f}^{\leq 2}(1) \otimes^{L} \mathbb{Z}_{X}(1) \stackrel{h_{2}}{\longrightarrow} \mathbb{Z}_{f}(\otimes) \rightarrow R^{\leq 2} f_{*} \mathbb{Z}_{Y}(1) \otimes^{L} \mathbb{Z}_{f}^{\leq 2}(1) \stackrel{+1}{\longrightarrow}
\end{aligned}
$$

et donc on a un diagramme commutatif de triangles (le lemme 3.7 ci-dessous) :

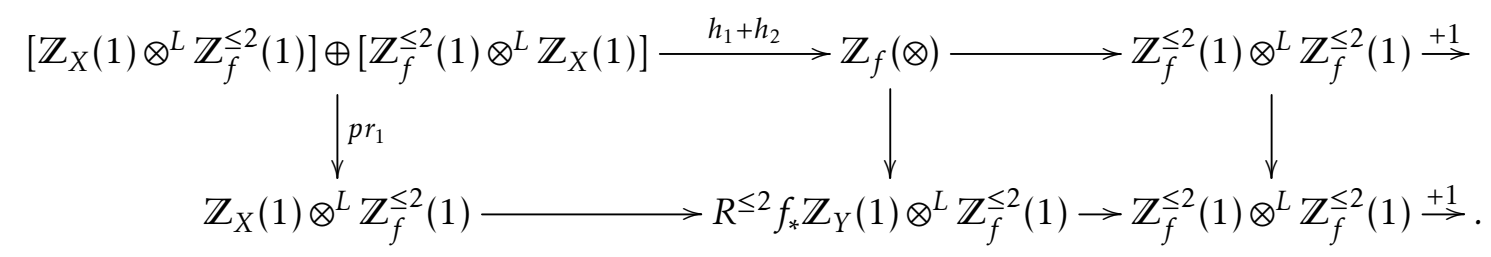

Notons $\mathbb{Z}(\wedge)$ le cône de $\mathbb{Z}_{X}(1) \otimes^{L} \mathbb{Z}_{f}^{\leq 2}(1) \stackrel{h_{\otimes} \circ h_{1}}{\longrightarrow} \mathbb{Z}_{f}(2)$, où $\mathbb{Z}_{f}(\otimes) \stackrel{h_{\otimes}}{\longrightarrow} \mathbb{Z}_{f}(2)$ est le morphisme du diagramme (3.10). Par les Propositions 3.1 et 3.5, on a $\tau^{\leq 3} \mathbb{Z}(\wedge) \cong \wedge^{2} T^{*}[-2]$.

Notons $\tau: \mathbb{Z}_{f}^{\leq 2}(1) \otimes L \mathbb{Z}_{X}(1) \rightarrow \mathbb{Z}_{X}(1) \otimes^{L} \mathbb{Z}_{f}^{\leq 2}(1)$ l'isomorphisme canonique. Puisque le produit $\mathbb{Z}(1) \otimes{ }^{L}$ $\mathbb{Z}(1) \rightarrow \mathbb{Z}(2)$ est symétrique, on a $h_{\otimes} \circ h_{1} \circ \tau=h_{\otimes} \circ h_{2}$ et un diagramme commutatif de triangles :

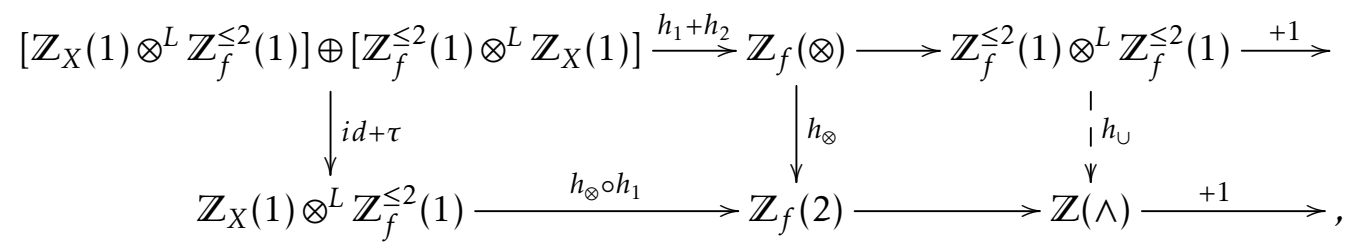

et l'homomorphisme $h$ est induit par le deuxième triangle. Puisque $\left[\mathbb{Z}_{f}^{\leq 2}(1) \otimes^{L} \mathbb{Z}_{f}^{\leq 2}(1)\right] \cong\left(T^{*} \otimes T^{*}\right)[-2]$, le morphisme $h_{\cup}$ induit un morphisme de faisceaux $\bar{k}$-constants : $T^{*} \otimes T^{*} \stackrel{h_{\cup}^{\prime}}{\longrightarrow} \wedge^{2} T^{*}$. On peut calculer $h_{\cup}^{\prime}$ en se ramenant au cas $Y \stackrel{\sim}{\rightarrow} X \times T$, et dans ce cas, $h_{\cup}^{\prime}$ est exactement le cup-produit $a \otimes b \rightarrow a \wedge b$. En général, $h_{\cup}^{\prime}$ est donc le cup-produit. Appliquons $\mathcal{H}^{2}(-)$ au diagrammes (3.12) et (3.13), on a un diagramme 
commutatif à lignes exactes

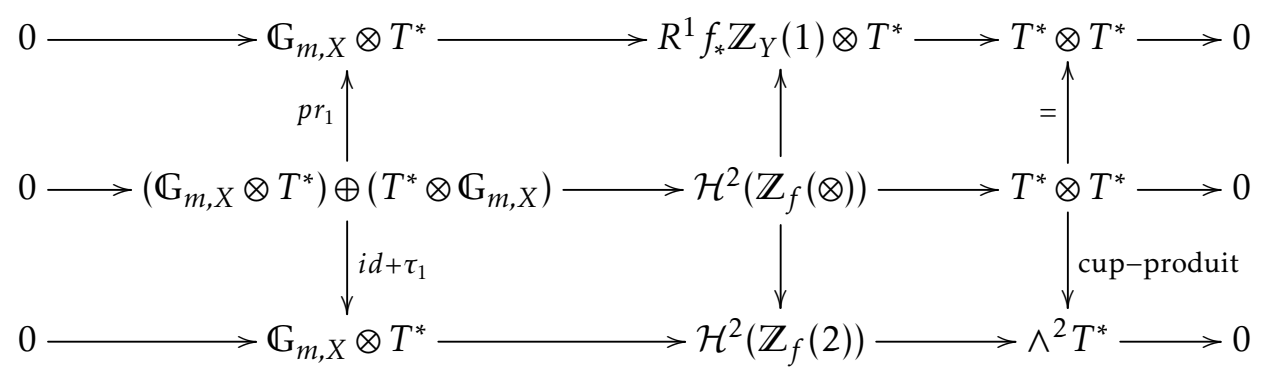

où la première ligne est $(3.5) \otimes T^{*}$, la troisième ligne est la suite exacte (3.11) et $\tau_{1}$ l'homomorphisme induit par $\tau$ en degré 1 , i.e.

$$
\tau_{1}: T^{*} \otimes \mathbb{G}_{m, X} \rightarrow \mathbb{G}_{m, X} \otimes T^{*}: t \otimes x \mapsto-x \otimes t .
$$

Appliquons $H^{i}\left(X_{\bar{k}},-\right)$ au diagramme ci-dessus, on a un diagramme commutatif :

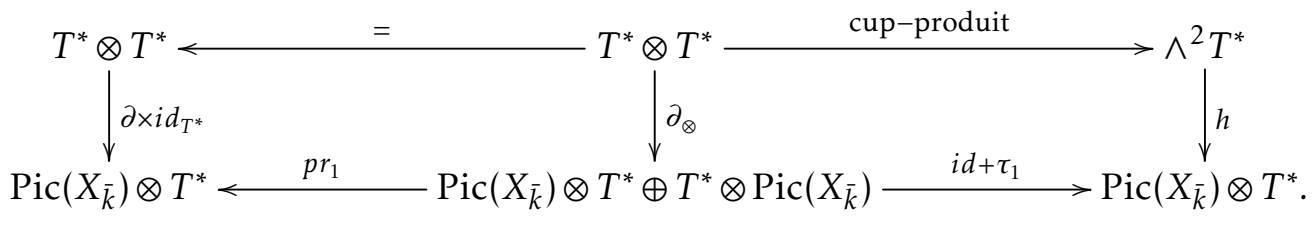

Donc $p r_{1}\left(\partial_{\otimes}(a \otimes b)\right)=\partial(a) \otimes b$. Par le même argument, $p r_{1}\left(\partial_{\otimes}(a \otimes b)\right)=a \otimes \partial(b)$. Ainsi on a : $\partial_{\otimes}(a \otimes b)=$ $(\partial(a) \otimes b, a \otimes \partial(b))$ et $h(a \wedge b)=a \otimes \partial(b)-b \otimes \partial(a)$.

Lemme 3.7. Soient $\mathcal{D}$ une catégorie triangulée et $A_{i} \stackrel{h_{i}}{\longrightarrow} B \stackrel{g_{i}}{\longrightarrow} C_{i} \stackrel{+1}{\longrightarrow}$ deux triangles pour $i=1,2$. Alors on a un diagramme commutatif de triangles :

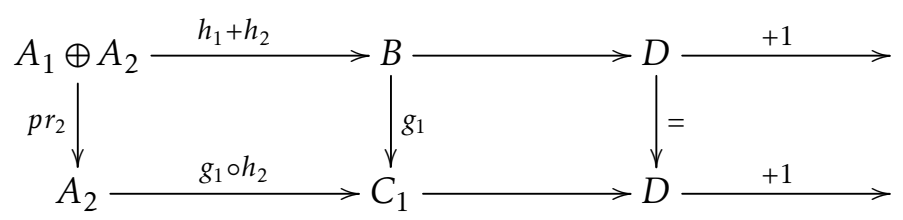

où $D$ est le cône de $\left(g_{1} \circ h_{2}\right)$.

Démonstration. Puisque Cone $\left(p r_{2}\right) \cong A_{1}[1] \cong \operatorname{Cone}\left(g_{1}\right)$, d'après [KS, Exer. 10.6], on a un diagramme commutatif de triangles :

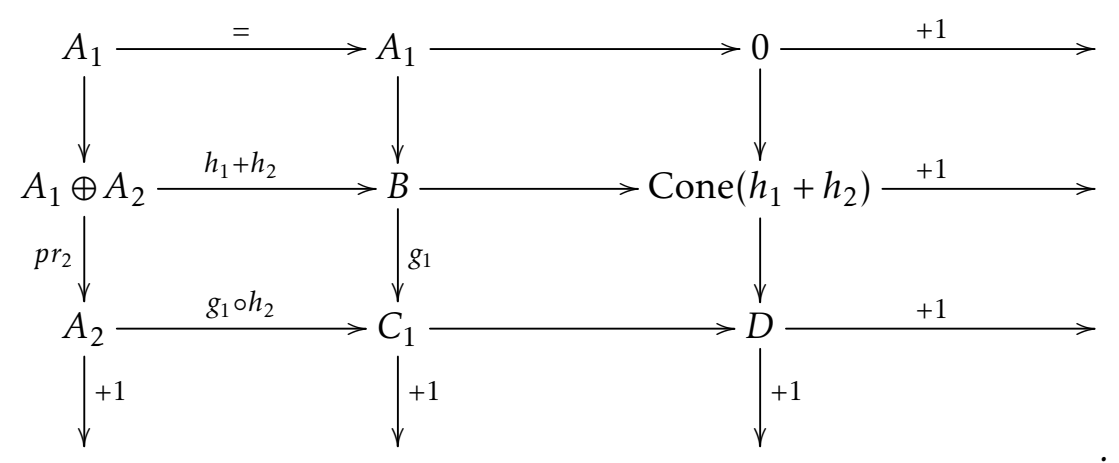

Donc Cone $\left(h_{1}+h_{2}\right) \cong \operatorname{Cone}\left(g_{1} \circ h_{2}\right) \cong D$, d'où le résultat. 


\section{Cohomologie motivique à coefficients $\mathbb{Z}(2)$ des torseurs universels sur une surface géométriquement rationnelle}

Soit $k$ un corps de caractéristique 0 . Soient $X$ une surface projective lisse géométriquement rationnelle sur $k$, et $g: \mathcal{T} \rightarrow X$ un torseur universel de $X$. Le résultat principal de cette section est le Théorème 4.7.

Lemme 4.1. Supposons que k est algébriquement clos. Soit X une k-variété projective, lisse, rationnelle, connexe. Soit $\mathcal{T} \rightarrow X$ un torseur universel sous le tore $T$. Soit $T^{c}$ une $T$-variété torique, projective, lisse. Soit $\mathcal{T}^{c}=\mathcal{T} \times{ }^{T} T^{c}$. Soit $Z$ une $T$-orbite de codimension $l$ dans $T^{c}$. Notons $\mathcal{Z}:=Z \times^{T} \mathcal{T} \subset \mathcal{T}^{c}$. Alors $\mathcal{Z}$ est lisse, $k[\mathcal{Z}]^{\times}=k^{\times}$, $\operatorname{Pic}(\mathcal{Z}) \cong \mathbb{Z}^{l}, \operatorname{Br}(\mathcal{Z})=0, H^{1}(\mathcal{Z}, \mathbb{Z} / n)=0, H_{\mathrm{nr}}^{2}(\mathcal{Z}, \mathbb{Q} / \mathbb{Z}(1))=0$ et $H^{2}(\mathcal{Z}, \mathbb{Z} / n(1)) \cong(\mathbb{Z} / n)^{l}$.

Démonstration. Notons $l^{\prime}:=\operatorname{dim}(T)-l$. Puisque $Z$ est une $T$-orbite de codimension $l$, par le Théorème 2.2, il y a une sous-variété torique affine $U \subset T^{c}$ telle que $U \cong \mathbb{A}^{l} \times \mathbb{G}_{m}^{l^{\prime}}$ comme variété torique et $Z \subset U$. Alors il y a un homomorphisme $\mathbb{G}_{m}^{l} \rightarrow T$ tel que $Z \stackrel{\sim}{\rightarrow} T / \mathbb{G}_{m}^{l}$. Notons $\phi: T \rightarrow T / \mathbb{G}_{m}^{l} \cong Z$. Alors $\mathcal{Z}:=\left(T / \mathbb{G}_{m}^{l}\right) \times^{T} \mathcal{T}$. Par la construction géométrique de $H^{1}(X, T) \stackrel{\phi_{*}}{\longrightarrow} H^{1}\left(X, T / \mathbb{G}_{m}^{l}\right)$, le morphisme $\mathcal{Z} \rightarrow X$ est un torseur sous $T / \mathbb{G}_{m}^{l}$ et $[\mathcal{Z}]=\phi_{*}([\mathcal{T}])$. Ainsi $\mathcal{Z}$ est lisse. Par $[\mathrm{CTS} 87, \S 2.1]$ (ou [S, Prop. 6.10]), on a un diagramme commutatif à lignes exactes :

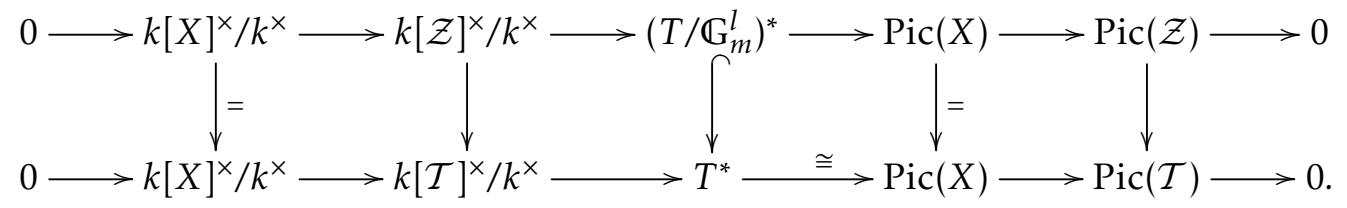

Puisque $\operatorname{Pic}(\mathcal{T})=0$ (Théorème 2.8), on a $k[\mathcal{Z}]^{\times}=k^{\times}$et $\operatorname{Pic}(\mathcal{Z}) \cong \mathbb{Z}^{l}$. Par [HS, Théorème 1.6] et le fait que $X$ est rationnelle, on a $\operatorname{Br}(\mathcal{Z})=0$. Ainsi $H_{\text {nr }}^{2}(\mathcal{Z}, \mathbb{Q} / \mathbb{Z}(1))=0$. Le reste du résultat se déduit de la suite de Kummer.

Lemme 4.2. Supposons que $k$ est algébriquement clos. Soient $X$ une $k$-variété projective, lisse, rationnelle et $\mathcal{T} \rightarrow X$ un torseur universel. Le morphisme naturel $H^{1}\left(X, \mathcal{K}_{2}\right) \rightarrow H^{1}\left(\mathcal{T}, \mathcal{K}_{2}\right)$ est nul.

Démonstration. On a un diagramme commutatif :

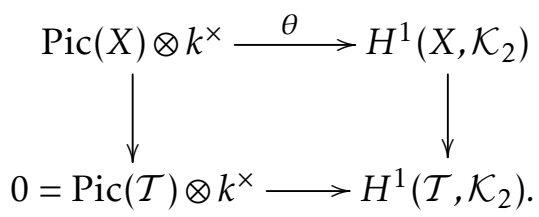

D'après [Pir, Prop. 2.6], le morphisme $\theta$ est un isomorphisme. On a $\operatorname{Pic}(\mathcal{T})=0$ par le Théorème 2.8. Donc $H^{1}\left(X, \mathcal{K}_{2}\right) \rightarrow H^{1}\left(\mathcal{T}, \mathcal{K}_{2}\right)$ est nul.

Théorème 4.3. Soit $X$ une $k$-variété projective, lisse, géométriquement intègre et géométriquement rationnelle. Soit $g: \mathcal{T} \rightarrow X$ un torseur universel de $X$. Alors on a $H_{\mathrm{nr}}^{3}\left(\mathcal{T}_{\bar{k}}, \mathbb{Q} / \mathbb{Z}(2)\right)=0$ et une suite exacte naturelle de $\Gamma_{k}$-modules :

$$
0 \rightarrow H^{1}\left(\mathcal{T}_{\bar{k}}, \mathcal{K}_{2}\right) \rightarrow \operatorname{Sym}^{2} \operatorname{Pic}\left(X_{\bar{k}}\right) \stackrel{\cup}{\rightarrow} \mathrm{CH}^{2}\left(X_{\bar{k}}\right) \rightarrow \mathrm{CH}^{2}\left(\mathcal{T}_{\bar{k}}\right) \rightarrow 0,
$$

où $\cup$ est induit par le produit d'intersection $\operatorname{Pic}\left(X_{\bar{k}}\right) \times \operatorname{Pic}\left(X_{\bar{k}}\right) \rightarrow \mathrm{CH}^{2}\left(X_{\bar{k}}\right)$.

Démonstration. Soient $T^{c}$ une $T$-variété torique, projective, lisse et $\mathcal{T}^{c}:=\mathcal{T} \times{ }^{T} T^{c}$. Puisque $X_{\bar{k}}$ est rationnelle, $H_{\mathrm{nr}}^{3}\left(X_{\bar{k}}, \mathbb{Q} / \mathbb{Z}(2)\right)=0$ et $\mathcal{T}_{\bar{k}}$ est rationnelle. Ainsi $H_{\mathrm{nr}}^{3}\left(\mathcal{T}_{\bar{k}}^{c}, \mathbb{Q} / \mathbb{Z}\right)=0$.

Par la résolution de Gersten, on a une suite exacte :

$$
0 \rightarrow H_{\mathrm{nr}}^{3}\left(\mathcal{T}_{\bar{k}}^{c}, \mathbb{Q} / \mathbb{Z}(2)\right) \rightarrow H_{\mathrm{nr}}^{3}\left(\mathcal{T}_{\bar{k}}, \mathbb{Q} / \mathbb{Z}(2)\right) \rightarrow \operatorname{ker}(\psi)
$$


où

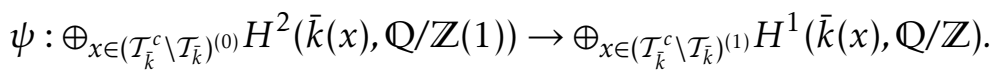

Soit $S_{1}$ l'ensemble des $T_{\bar{k}}$-orbites de codimension 1 de $T_{\bar{k}}^{c}$. Pour $Z \in S_{1}$, notons $\mathcal{Z}:=Z \times^{T} \mathcal{T} \subset \mathcal{T}^{c}$. Alors $S_{1} \cong\left(\mathcal{T}_{\bar{k}}^{c} \backslash \mathcal{T}_{\bar{k}}\right)^{(0)}$ et

$$
\operatorname{ker}(\psi) \subset \oplus_{Z \in S_{1}} H_{\mathrm{nr}}^{2}(\mathcal{Z}, \mathbb{Q} / \mathbb{Z}(1)) \subset \oplus_{x \in\left(\mathcal{T}_{\bar{k}}^{c} \backslash \mathcal{T}_{\bar{k}}\right)^{(0)}} H^{2}(\bar{k}(x), \mathbb{Q} / \mathbb{Z}(1))
$$

D'après le Lemme $4.1, \operatorname{ker}(\psi)=0$, et donc $H_{\mathrm{nr}}^{3}\left(\mathcal{T}_{\bar{k}}, \mathbb{Q} / \mathbb{Z}(2)\right)=0$.

D'après le Théorème 3.6,

$$
\mathbb{H}^{3}\left(X_{\bar{k}}, \mathbb{Z}_{g}(2)\right) \cong T^{*} \otimes T^{*} / \wedge^{2} T^{*} \cong \operatorname{Sym}^{2} T^{*} \cong \operatorname{Sym}^{2} \operatorname{Pic}\left(X_{\bar{k}}\right) .
$$

Par le Théorème $2.1, \mathrm{CH}^{2}\left(X_{\bar{k}}\right) \cong \mathbb{H}^{4}\left(X_{\bar{k}}, \mathbb{Z}(2)\right)$ et $\mathrm{CH}^{2}\left(\mathcal{T}_{\bar{k}}\right) \cong \mathbb{H}^{4}\left(\mathcal{T}_{\bar{k}}, \mathbb{Z}(2)\right)$. Pour tout $x \in X_{\bar{k}}$, la fibre $\mathcal{T}_{\bar{k}, x} \cong \mathbb{G}_{m, k(x)}^{\operatorname{dim}(T)}$ et donc $\mathrm{CH}^{i}\left(\mathcal{T}_{\bar{k}, x}\right)=0$ pour tout $i \geq 1$. D'après [Ro96, Cor. 8.2], le morphisme de restriction $\mathrm{CH}^{2}\left(X_{\bar{k}}\right) \rightarrow \mathrm{CH}^{2}\left(\mathcal{T}_{\bar{k}}\right)$ est surjectif. On conclut alors avec le Lemme 4.2 et le Théorème 3.6.

Corollaire 4.4. Sous les hypothèses $d u$ Théorème 4.3, soient $U \subset X$ un ouvert et $\mathcal{T}_{U}:=\mathcal{T} \times_{X} U$. Supposons que $\operatorname{codim}(X \backslash U, X) \geq 2$ et $H^{1}\left(X_{\bar{k}}, \mathcal{K}_{2}\right) \cong H^{1}\left(U_{\bar{k}}, \mathcal{K}_{2}\right)$. Alors on a $H_{\mathrm{nr}}^{3}\left(\mathcal{T}_{U, \bar{k}}, \mathbb{Q} / \mathbb{Z}(2)\right)=0$ et une suite exacte naturelle de $\Gamma_{k}$-modules :

$$
0 \rightarrow H^{1}\left(\mathcal{T}_{U, \bar{k}}, \mathcal{K}_{2}\right) \rightarrow \operatorname{Sym}^{2} \operatorname{Pic}\left(U_{\bar{k}}\right) \rightarrow \mathrm{CH}^{2}\left(U_{\bar{k}}\right) \rightarrow \mathrm{CH}^{2}\left(\mathcal{T}_{U, \bar{k}}\right) \rightarrow 0 .
$$

Démonstration. Puisque la cohomologie non ramifiée ne dépend pas des sous-ensembles de codimension $\geq 2$, on a $H_{\mathrm{nr}}^{3}\left(\mathcal{T}_{U, \bar{k}}, \mathbb{Q} / \mathbb{Z}(2)\right)=0$ et $H_{\mathrm{nr}}^{3}\left(U_{\bar{k}}, \mathbb{Q} / \mathbb{Z}(2)\right)=0$. Par le Théorème $2.1, \mathrm{CH}^{2}\left(U_{\bar{k}}\right) \cong \mathbb{H}^{4}\left(U_{\bar{k}}, \mathbb{Z}(2)\right)$ et $\mathrm{CH}^{2}\left(\mathcal{T}_{U, \bar{k}}\right) \cong \mathbb{H}^{4}\left(\mathcal{T}_{U, \bar{k}}, \mathbb{Z}(2)\right)$. D'après le Théorème 3.6, on a un diagramme commutatif de suites exactes :

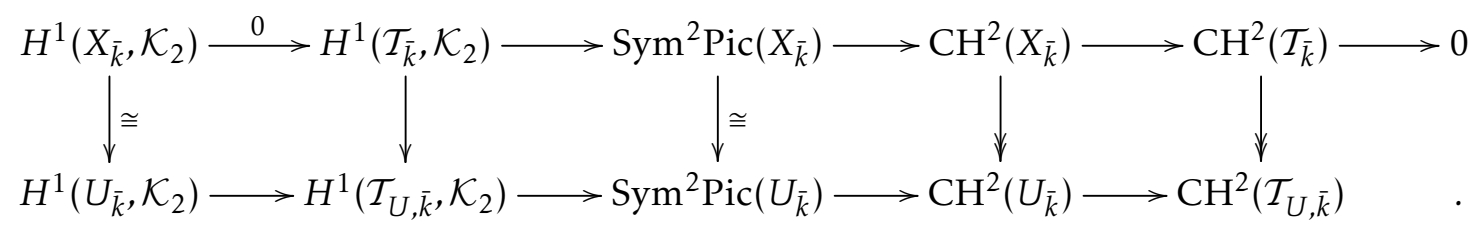

Le résultat en découle.

Proposition 4.5. Supposons que $k$ est algébriquement clos. Soient X une $k$-surface projective lisse rationnelle et $\mathcal{T}$ un torseur universel sur X. Alors $\mathrm{CH}^{2}(\mathcal{T})=0$.

Démonstration. On a un diagramme commutatif :

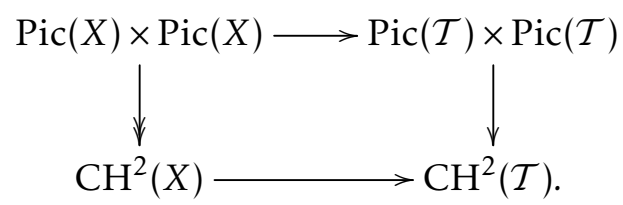

D'après le Théorème 4.3, le morphisme $\mathrm{CH}^{2}(X) \rightarrow \mathrm{CH}^{2}(\mathcal{T})$ est surjectif. Donc le morphisme $\operatorname{Pic}(\mathcal{T}) \times$ $\operatorname{Pic}(\mathcal{T}) \rightarrow \mathrm{CH}^{2}(\mathcal{T})$ est surjectif. Puisque $\operatorname{Pic}(\mathcal{T})=0$, on a $\mathrm{CH}^{2}(\mathcal{T})=0$.

Une conséquence immédiate du Théorème 4.3 et de la Proposition 4.5 est :

Corollaire 4.6. Soient $X$ une $k$-surface projective lisse géométriquement rationnelle et $\mathcal{T}$ un torseur universel sur $X$. On a alors une suite exacte naturelle de réseaux galoisiens

$$
0 \rightarrow H^{1}\left(\mathcal{T}_{\bar{k}}, \mathcal{K}_{2}\right) \rightarrow \operatorname{Sym}^{2} \operatorname{Pic}\left(X_{\bar{k}}\right) \stackrel{\cup}{\rightarrow} \mathbb{Z} \rightarrow 0 .
$$


Théorème 4.7. Soient $X$ une $k$-surface projective lisse géométriquement rationnelle et $\mathcal{T}$ un torseur universel sur X. Alors

(1) Les groupes $\mathbb{H}^{4}(\mathcal{T}, \mathbb{Z}(2)) / \mathbb{H}^{4}(k, \mathbb{Z}(2)), \frac{H_{\mathrm{nr}}^{3}(\mathcal{T}, \mathbb{Q} / \mathbb{Z}(2))}{H^{3}(k, \mathbb{Q} / \mathbb{Z}(2))}$ et $\mathrm{CH}^{2}(\mathcal{T})$ sont finis;

(2) On a des suites exactes

$$
0 \rightarrow \mathbb{H}^{4}(\mathcal{T}, \mathbb{Z}(2)) / \mathbb{H}^{4}(k, \mathbb{Z}(2)) \rightarrow H^{1}\left(k, H^{1}\left(\mathcal{T}_{\bar{k}}, \mathcal{K}_{2}\right)\right) \rightarrow \operatorname{Ker}\left(\mathbb{H}^{5}(k, \mathbb{Z}(2)) \rightarrow \mathbb{H}^{5}(\mathcal{T}, \mathbb{Z}(2))\right)
$$

et

$$
\left(\operatorname{Sym}^{2} \operatorname{Pic}\left(X_{\bar{k}}\right)\right)^{\Gamma_{k}} \stackrel{\cup}{\rightarrow} \mathbb{Z} \cong \mathrm{CH}^{2}\left(X_{\bar{k}}\right) \rightarrow H^{1}\left(k, H^{1}\left(\mathcal{T}_{\bar{k}}, \mathcal{K}_{2}\right)\right) \rightarrow H^{1}\left(k, \operatorname{Sym}^{2} \operatorname{Pic}\left(X_{\bar{k}}\right)\right) \rightarrow 0 ;
$$

(3) $\operatorname{Si~} H^{1}\left(k, \operatorname{Sym}^{2} \operatorname{Pic}\left(X_{\bar{k}}\right)\right)=0$ et $X(k) \neq \emptyset$, alors $\frac{H_{\mathrm{nr}}^{3}(\mathcal{T}, \mathbb{Q} / \mathbb{Z}(2))}{H^{3}(k, \mathbb{Q} / \mathbb{Z}(2))}=0$.

Démonstration. Par la Proposition 4.5 et le Théorème $4.3, \mathrm{CH}^{2}\left(\mathcal{T}_{\bar{k}}\right)=0, \mathbb{H}^{4}\left(\mathcal{T}_{\bar{k}}, \mathbb{Z}(2)\right)=0$ et $H^{1}\left(\mathcal{T}_{\bar{k}}, \mathcal{K}_{2}\right)$ est de type fini sans torsion. Ainsi $H^{1}\left(k, H^{1}\left(\mathcal{T}_{\bar{k}}, \mathcal{K}_{2}\right)\right)$ est fini. Par $[\mathrm{CT} 15, \S 2.2]$ et le fait que $H^{0}\left(\mathcal{T}_{\bar{k}}, \mathcal{K}_{2}\right)$ est uniquement divisible (Corollaire 2.9), on a une suite exacte :

$$
\mathbb{H}^{4}(k, \mathbb{Z}(2)) \rightarrow \mathbb{H}^{4}(\mathcal{T}, \mathbb{Z}(2)) \rightarrow H^{1}\left(k, H^{1}\left(\mathcal{T}_{\bar{k}}, \mathcal{K}_{2}\right)\right) \rightarrow \operatorname{Ker}\left(\mathbb{H}^{5}(k, \mathbb{Z}(2)) \rightarrow \mathbb{H}^{5}(\mathcal{T}, \mathbb{Z}(2))\right) .
$$

La première suite exacte de (2) en découle. Par la suite exacte (2.3), les groupes $\frac{H_{\text {nn }}^{3}(\mathcal{T}, \mathbb{Q} / \mathbb{Z}(2))}{H^{3}(k, \mathbb{Q} / \mathbb{Z}(2))}$ et $\mathrm{CH}^{2}(\mathcal{T})$ sont finis.

Puisque $\mathbb{H}^{4}\left(X_{\bar{k}}, \mathbb{Z}(2)\right) \cong \mathrm{CH}^{2}\left(X_{\bar{k}}\right) \cong \mathbb{Z}$, on a $H^{1}\left(k, H^{4}\left(X_{\bar{k}}, \mathbb{Z}(2)\right)\right)=0$. Une application du Corollaire 4.6 donne la deuxière suite exacte de (2).

Soit $F \subset \mathcal{T}$ un sous-ensemble fermé de codimension $\geq 2$. Alors $\mathrm{CH}^{2}\left((\mathcal{T} \backslash F)_{\bar{k}}\right)=0$,

$$
H_{\mathrm{nr}}^{3}\left((\mathcal{T} \backslash F)_{\bar{k}}, \mathbb{Q} / \mathbb{Z}(2)\right) \cong H_{\mathrm{nr}}^{3}\left(\mathcal{T}_{\bar{k}}, \mathbb{Q} / \mathbb{Z}(2)\right)=0 \quad \text { et donc } \quad H^{4}\left((\mathcal{T} \backslash F)_{\bar{k}}, \mathbb{Z}(2)\right)=0 .
$$

Par la résolution de Gersten (2.1), on a un isomorphisme entre $H^{0}\left(\mathcal{T}_{\bar{k}}, \mathcal{K}_{2}\right)$ et $H^{0}\left((\mathcal{T} \backslash F)_{\bar{k}}, \mathcal{K}_{2}\right)$ et donc $H^{0}\left((\mathcal{T} \backslash F)_{\bar{k}}, \mathcal{K}_{2}\right)$ est uniquement divisible. D'après [CT15, §2.2], la suite exacte (4.2) vaut aussi pour $\mathcal{T} \backslash F$. Donc on a un morphisme naturel injectif :

$$
\mathbb{H}^{4}(\mathcal{T} \backslash F, \mathbb{Z}(2)) / \mathbb{H}^{4}(k, \mathbb{Z}(2)) \hookrightarrow H^{1}\left(k, H^{1}\left((\mathcal{T} \backslash F)_{\bar{k}}, \mathcal{K}_{2}\right)\right),
$$

qui est un isomorphisme si $(\mathcal{T} \backslash F)(k) \neq \emptyset$.

Sous les hypothèses de (3), soient $x \in X(k), U:=X \backslash\{x\}$ et $\mathcal{T}_{U}:=\mathcal{T} \times_{X} U$. Alors $\operatorname{codim}\left(\mathcal{T} \backslash \mathcal{T}_{U}, \mathcal{T}\right) \geq 2$. Par la résolution de Gersten (2.1), on a une suite exacte :

$$
0 \rightarrow H^{1}\left(X_{\bar{k}}, \mathcal{K}_{2}\right) \rightarrow H^{1}\left(U_{\bar{k}}, \mathcal{K}_{2}\right) \rightarrow \mathbb{Z} \cdot x \stackrel{\phi}{\rightarrow} \mathrm{CH}^{2}\left(X_{\bar{k}}\right) \rightarrow \mathrm{CH}^{2}\left(U_{\bar{k}}\right) \rightarrow 0
$$

Puisque $\phi$ est un isomorphisme, on a $H^{1}\left(X_{\bar{k}}, \mathcal{K}_{2}\right) \cong H^{1}\left(U_{\bar{k}}, \mathcal{K}_{2}\right)$ et $\mathrm{CH}^{2}\left(U_{\bar{k}}\right)=0$. D'après le Corollaire 4.4, $H^{1}\left(\mathcal{T}_{U, \bar{k}}, \mathcal{K}_{2}\right) \cong \operatorname{Sym}^{2} \operatorname{Pic}\left(X_{\bar{k}}\right)$. Par hypothèses, $H^{1}\left(k, H^{1}\left(\mathcal{T}_{U, \bar{k}}, \mathcal{K}_{2}\right)\right)=0$. D'après (4.3), le groupe $\mathbb{H}^{4}\left(\mathcal{T}_{U}, \mathbb{Z}(2)\right) / \mathbb{H}^{4}(k, \mathbb{Z}(2))$ est trivial. Une application du fait $H_{\mathrm{nr}}^{3}(\mathcal{T}, \mathbb{Q} / \mathbb{Z}(2)) \cong H_{\mathrm{nr}}^{3}\left(\mathcal{T}_{U}, \mathbb{Q} / \mathbb{Z}(2)\right)$ donne (3).

Remarque 4.8. Dans le Théorème 4.7, le critère $H^{1}\left(k, \operatorname{Sym}^{2} \operatorname{Pic}\left(X_{\bar{k}}\right)\right)=0$ n'est pas birationnellement invariant. En fait, soit $X$ une surface projective lisse géométriquement rationnelle avec $X(k) \neq \emptyset$ et $X^{\prime}$ un éclatement de $X$ en un $k$-point. Supposons $H^{1}\left(k, \operatorname{Pic}\left(X_{\bar{k}}\right)\right) \neq 0$ (cf. [Ma, §31] pour des exemples). Alors $\operatorname{Pic}\left(X_{\bar{k}}^{\prime}\right) \stackrel{\sim}{\rightarrow} \operatorname{Pic}\left(X_{\bar{k}}\right) \oplus \mathbb{Z}$ et

$$
H^{1}\left(k, \operatorname{Sym}^{2} \operatorname{Pic}\left(X_{\bar{k}}^{\prime}\right)\right) \cong H^{1}\left(k, \operatorname{Sym}^{2} \operatorname{Pic}\left(X_{\bar{k}}\right) \oplus \operatorname{Pic}\left(X_{\bar{k}}\right) \oplus \mathbb{Z}\right) .
$$

Alors, même si $H^{1}\left(k, \operatorname{Sym}^{2} \operatorname{Pic}\left(X_{\bar{k}}\right)\right)=0$, on a $H^{1}\left(k, \operatorname{Sym}^{2} \operatorname{Pic}\left(X_{\bar{k}}^{\prime}\right)\right) \neq 0$. 
Corollaire 4.9. Sous les hypothèses du Théorème 4.7, le morphisme canonique $H^{3}(\mathcal{T}, \mathbb{Q} / \mathbb{Z}(2)) \rightarrow H_{\mathrm{nr}}^{3}(\mathcal{T}, \mathbb{Q} / \mathbb{Z}(2))$ est surjectif.

Démonstration. Par [CT91, Suite 3.10], on a une suite exacte :

$$
0 \rightarrow N H^{3}(\mathcal{T}, \mathbb{Q} / \mathbb{Z}(2)) \rightarrow H^{3}(\mathcal{T}, \mathbb{Q} / \mathbb{Z}(2)) \rightarrow H_{\mathrm{nr}}^{3}(\mathcal{T}, \mathbb{Q} / \mathbb{Z}(2)) \rightarrow \mathrm{CH}^{2}(\mathcal{T}) \otimes \mathbb{Q} / \mathbb{Z} .
$$

Par le Théorème 4.7, $\mathrm{CH}^{2}(\mathcal{T})$ est fini, et donc $\mathrm{CH}^{2}(\mathcal{T}) \otimes \mathbb{Q} / \mathbb{Z}=0$. Donc le morphisme $H^{3}(\mathcal{T}, \mathbb{Q} / \mathbb{Z}(2)) \rightarrow$ $H_{\mathrm{nr}}^{3}(\mathcal{T}, \mathbb{Q} / \mathbb{Z}(2))$ est surjectif.

\section{Surfaces de Châtelet généralisées}

Dans cette section, $X$ est une surface de Châtelet généralisée, c'est-à-dire un fibré en coniques sur $\mathbb{P}_{k}^{1}$ possédant une section sur une extension quadratique $k^{\prime}$ de $k$. Soient $\mathcal{T}$ un torseur universel de $X$ et $\mathcal{T}^{c}$ une compactification lisse de $\mathcal{T}$. Alors $X_{k^{\prime}}$ est $k^{\prime}$-rationnel et, par le corollaire $2.10, \frac{H_{\text {nrl }}^{3}\left(\mathcal{T}^{c}, \mathbb{Q} / \mathbb{Z}(2)\right)}{H^{3}(k, \mathbb{Q} / \mathbb{Z}(2))}$ est annulé par 2.

Le résultat principal de ce paragraphe est :

Théorème 5.1. Soient $X$ une variété lisse projective, $P(t)$ est un polynôme séparable et supposons que $X$ contienne un ouvert de la forme Spec $k[y, z, t] /\left(y^{2}-a z^{2}=P(t)\right)$. Soient $\mathcal{T}$ un torseur universel de $X$ et $\mathcal{T}^{c}$ une compactification lisse de $\mathcal{T}$. Supposons que $X(k) \neq \emptyset$. Alors $\frac{H_{\mathrm{nr}}^{3}\left(\mathcal{T}^{c}, \mathbb{Q} / \mathbb{Z}(2)\right)}{H^{3}(k, \mathbb{Q} / \mathbb{Z}(2))}=0$.

Dans ce théorème, $P(t)$ est un polynôme séparable de degré quelconque (dans la définition des surfaces de Châtelet généralisées dans [CT87], $P(t)$ est de degré 3 ou 4).

Démonstration. Si $X$ et $X_{1}$ sont birationnellement équivalents, alors $X_{1}(k) \neq \emptyset$ et il existe un torseur universel $\mathcal{T}_{1}^{c}$ de $X_{1}$ tel que $\mathcal{T}^{c}$ et $\mathcal{T}_{1}^{c}$ soient stablement birationnellement équivalents par [CTS87, Prop. 2.9.2]. Donc on a $H_{\mathrm{nr}}^{3}\left(\mathcal{T}^{c}, \mathbb{Q} / \mathbb{Z}(2)\right) \stackrel{\sim}{\rightarrow} H_{\mathrm{nr}}^{3}\left(\mathcal{T}_{1}^{c}, \mathbb{Q} / \mathbb{Z}(2)\right)$. C'est-à-dire que l'on peut remplacer $X$ par $X_{1}$. Ainsi, comme dans [Sko, p. 135], on peut supposer que $X$ est une variété qui vérifie les conditions de la Proposition 5.2 ci-dessous. Par la Proposition 5.2, on a $H^{1}\left(k, \operatorname{Sym}^{2} \operatorname{Pic}\left(X_{\bar{k}}\right)\right)=0$. D'après le Théorème 4.7, on a $\frac{H_{\mathrm{nr}}^{3}\left(\mathcal{T}^{c}, \mathbb{Q} / \mathbb{Z}(2)\right)}{H^{3}(k, \mathbb{Q} / \mathbb{Z}(2))}=0$

Proposition 5.2. Soient $P(t) \in k[t]$ un polynôme séparable de degré pair, $\left(P_{i}(t)\right)_{i \in I}$ ses facteurs irréductibles, et $a \in k$ un élément tel que a n'est un carré de $k[t] / P_{i}(t)$ pour aucun $i \in I$ (i.e. chaque $P_{i}(t)$ est irréductible dans $k(\sqrt{a})[t])$. Soit $X_{1}$ (resp. $\left.X_{2}\right)$ une sous-variété fermée de $\mathrm{P}^{2} \times\left(\mathrm{P}^{1} \backslash \infty\right)\left(\right.$ resp. de $\left.\mathrm{P}^{2} \times\left(\mathrm{P}^{1} \backslash 0\right)\right)$ avec les coordonnées $(y: z: u ; t)\left(\right.$ resp. $\left.\left(y^{\prime}: z^{\prime}: u^{\prime} ; t^{\prime}\right)\right)$ donnée par $y^{2}-a z^{2}-P(t) u^{2}=0\left(\right.$ resp. $\left.\left(y^{\prime}\right)^{2}-a\left(z^{\prime}\right)^{2}-P\left(\left(t^{\prime}\right)^{-1}\right)\left(u^{\prime}\right)^{2}=0\right)$. On recolle $X_{1}$ et $X_{2}$ sur $\mathrm{P}^{1} \backslash(0 \cup \infty)$ par $t=\left(t^{\prime}\right)^{-1}, y=y^{\prime}, z=z^{\prime}$ et $u=\left(t^{\prime}\right)^{n / 2} u^{\prime}$, et on la note par $X$. Notons $T^{*}:=\operatorname{Pic}\left(X_{\bar{k}}\right)$. Alors on a $H^{1}\left(k, \operatorname{Sym}^{2} T^{*}\right)=0$.

Notons $k^{\prime}:=k(\sqrt{a})$. Soient $\sigma \in \operatorname{Gal}\left(k^{\prime} / k\right)$ l'élément non-trivial, $\left(P_{i}(t)\right)_{i \in I}$ les facteurs irréductibles de $P(t)$, et $\left(e_{j_{i}}\right)_{j_{i} \in J_{i}}$ les racines de $P_{i}(t)$ dans $\bar{k}$. Soient $O_{i, i^{\prime}}$ l'ensemble des $\Gamma_{k^{\prime}}$-orbites de $J_{i} \times J_{i^{\prime}}$ si $i \neq i^{\prime}$, et $O_{i, i}$ l'ensemble des $\Gamma_{k^{\prime}}$-orbites de $J_{i} \times J_{i} / \sim$, où $\sim$ est donné par $\left(j_{i}, j_{i}^{\prime}\right) \sim\left(j_{i}^{\prime}, j_{i}\right)$. Soit $\Delta_{i}$ l'orbite diagonale de $O_{i, i}$. Pour une orbite $S$, on note $|S|$ le nombre des éléments de $S$. L'action de $\Gamma_{k}$ sur $J_{i}$ et $J_{i}$, induit une action de $\sigma$ sur $O_{i, i^{\prime}}$. Ainsi $\sigma\left(\Delta_{i}\right)=\Delta_{i}$.

Lemme 5.3. Si $\left|J_{i_{0}}\right|$ est impair, alors il existe au moins une orbite $S \in O_{i_{0}, i}$ pour chaque $i \neq i_{0}$, telle que $\sigma(S)=S$.

Démonstration. Puisque l'action de $\Gamma_{k^{\prime}}$ sur $J_{i}$ est transitive, pour chaque orbite $S \in O_{i_{0}, i},|S|$ est divisible par $\left|J_{i}\right|$. Donc si $S \neq \sigma(S),|S \cup \sigma(S)|$ est divisible par $2 \cdot\left|J_{i}\right|$. Puisque $\left|J_{i_{0}} \times J_{i}\right|=\left|J_{i_{0}}\right| \cdot\left|J_{i}\right|$ et $\left|J_{i_{0}}\right|$ est impair, il existe au moins une orbite $S \in O_{i_{0}, i}$ telle que $\sigma(S)=S$. 
Lemme 5.4. Pour chaque $i$ et chaque orbite $S \in O_{i, i}$, on a $2 \cdot|S|$ est divisible par $\left|J_{i}\right|$. De plus, si $\left|J_{i}\right|$ est pair, alors il existe au moins une orbite $S \in O_{i, i}$, telle que $\sigma(S)=S$ et $|S|=s \cdot\left|J_{i}\right| / 2$, où $S$ est un entier impair.

Démonstration. Soit $\left(j_{i}, j_{i}^{\prime}\right)$ un élément de l'orbite $S \in O_{i, i}$. Pour l'action de $\Gamma_{k^{\prime}}$, soit $S t_{j}\left(\right.$ resp. $\left.S t_{j, j^{\prime}}\right)$ le stabilisateur de $j_{i}\left(\operatorname{resp}\right.$. de $\left.\left(j_{i}, j_{i}^{\prime}\right)\right)$. Ainsi s'il y a $g \in \Gamma_{k^{\prime}}$ tel que $g\left(j_{i}\right)=j_{i}^{\prime}$ et $g\left(j_{i}^{\prime}\right)=j_{i}$, alors

$S t_{j, j^{\prime}}=\left(S t_{j} \cap\left(g \cdot S t_{j} \cdot g^{-1}\right)\right) \cup\left(\left(g \cdot S t_{j}\right) \cap\left(g \cdot\left(g \cdot S t_{j} \cdot g^{-1}\right)\right)\right)=\left(S t_{j} \cap\left(g \cdot S t_{j} \cdot g^{-1}\right)\right) \cup\left(g \cdot\left(S t_{j} \cap\left(g \cdot S t_{j} \cdot g{ }^{-1}\right)\right)\right)$;

sinon, $S t_{j, j^{\prime}}$ est un sous-groupe de $S t_{j}$. Ainsi $2 \cdot\left[\Gamma_{k^{\prime}}: S t_{j, j^{\prime}}\right]$ est divisible par $\left[\Gamma_{k^{\prime}}: S t_{j}\right]$, et donc $2 \cdot|S|$ est divisible par $\left|J_{i}\right|$.

Supposons que $\left|J_{i}\right|$ est pair. On a $\left|J_{i} \times J_{i} / \sim\right|=\left|J_{i}\right|\left|J_{i}-1\right| / 2$. D'après l'argument du Lemme 5.3 , il existe au moins une orbite $S \in O_{i, i}$, telle que $\sigma(S)=S$ et $|S|=s \cdot\left|J_{i}\right| / 2$, où $s$ est un entier impair.

Démonstration de la Proposition 5.2. Soient $F$ le diviseur de $X_{\bar{k}}$ défini par $t=\infty$, $G$ le diviseur de $X_{\bar{k}}$ défini $\operatorname{par}(u, y-\sqrt{a} z), G^{\prime}$ le diviseur de $X_{\bar{k}}$ défini par $(u, y+\sqrt{a} z), D_{j_{i}}$ le diviseur de $X_{\bar{k}}$ défini par $\left(t-e_{j_{i}}, y-\sqrt{a} z\right)$ et $D_{j_{i}}^{\prime}$ le diviseur de $X_{\bar{k}}$ défini par $\left(t-e_{j_{i}}, y+\sqrt{a} z\right)$, pour chaque $i \in I$ et chaque $j_{i} \in J_{i}$. Pour chaque $i \in I$, on note $D_{i}:=\sum_{j_{i} \in J_{i}} D_{j_{i}}$ et $D_{i}^{\prime}:=\sum_{j_{i} \in J_{i}} D_{j_{i}}^{\prime}$. Pour chaque orbite $S \in O_{i, i^{\prime}}$, on note

$$
D_{S}:=\sum_{\left(j_{i}, j_{i^{\prime}}\right) \in S} D_{j_{i}} \cdot D_{j_{i^{\prime}}} \in \operatorname{Sym}{ }^{2} \operatorname{Pic}\left(X_{\bar{k}}\right) \text { et } D_{S}^{\prime}:=\sum_{\left(j_{i}, j_{i^{\prime}}\right) \in S} D_{j_{i}}^{\prime} \cdot D_{j_{i^{\prime}}}^{\prime} \in \operatorname{Sym}{ }^{2} \operatorname{Pic}\left(X_{\bar{k}}\right) \text {. }
$$

D’après la démonstration de [CTCS, Prop. 5.1] (cf. [CTSSD, Section 7] pour plus de détails dans le cas où $\operatorname{deg}(P)=4), T^{*}=\operatorname{Pic}\left(X_{\bar{k}}\right)$ est engendré par $\left(D_{j_{i}}\right)_{j_{i} \in \cup_{i} J_{i}},\left(D_{j_{i}}^{\prime}\right)_{j_{i} \in \cup_{i} J_{i}}, F, G$ et $G^{\prime}$, et les relations sont : $G^{\prime}-G=\sum_{i} \sum_{j_{i}} D_{j_{i}}-\frac{\sum_{i}\left|J_{i}\right|}{2} F$ et $D_{j_{i}}+D_{j_{i}}^{\prime}=F$ pour chaque $j_{i}$. Ainsi $\operatorname{Pic}\left(X_{\bar{k}}\right)$ est un $\mathbb{Z}$-module libre de base $\left(D_{j_{i}}\right)_{j_{i} \in \cup_{i} J_{i}}, F$ et $G$, et l'action de $\Gamma_{k^{\prime}}$ sur cette base est de permutation. Donc l'action de $\Gamma_{k^{\prime}} \operatorname{sur} \mathrm{Sym}^{2} T^{*}$ est aussi de permutation. Ainsi $H^{1}\left(k^{\prime}, \operatorname{Sym}^{2} T^{*}\right)=0$. Par la suite spectrale de Hochschild-Serre, on a :

$$
H^{1}\left(\operatorname{Gal}\left(k^{\prime} / k\right),\left(\operatorname{Sym}^{2} T^{*}\right)^{\Gamma_{k^{\prime}}}\right) \cong H^{1}\left(k, \operatorname{Sym}^{2} T^{*}\right) \text {. }
$$

Ainsi il suffit de montrer que $H^{1}\left(\operatorname{Gal}\left(k^{\prime} / k\right),\left(\operatorname{Sym}^{2} T^{*}\right)^{\Gamma_{k^{\prime}}}\right)=0$.

L'idée de la démonstration de l'égalité $H^{1}\left(\operatorname{Gal}\left(k^{\prime} / k\right),\left(\operatorname{Sym}^{2} T^{*}\right)^{\Gamma_{k^{\prime}}}\right)=0$ est de trouver une filtration $\operatorname{Gal}\left(k^{\prime} / k\right)$-invariante de $\left(\operatorname{Sym}^{2} T^{*}\right)^{\Gamma_{k^{\prime}}}$ telle que la cohomologie des quotients successifs est 0 . En fait, on trouve des sous-groupes $A_{l} \subset\left(\operatorname{Sym}^{2} T^{*}\right)^{\Gamma_{k^{\prime}}}, l=1, \ldots, m$, tels que $\left(\operatorname{Sym}^{2} T^{*}\right)^{\Gamma_{k^{\prime}}} \stackrel{\sim}{\rightarrow} \bigoplus_{l} A_{l}$, le sous-groupe $\bigoplus_{l=1}^{l_{0}} A_{l} \subset\left(\operatorname{Sym}^{2} T^{*}\right)^{\Gamma_{k^{\prime}}}$ soit $\operatorname{Gal}\left(k^{\prime} / k\right)$-invariant, et $H^{1}\left(k, A_{l_{0}}^{\prime}\right)=0$ pour tous les $l_{0}=1, \ldots, m$, où $A_{l_{0}}^{\prime}:=$ $\bigoplus_{l=1}^{l_{0}} A_{l} / \bigoplus_{l=1}^{l_{0}-1} A_{l}$.

Puisque $\operatorname{Pic}\left(X_{\bar{k}}\right)$ est un $\mathbb{Z}$-module libre de base $\left(D_{j_{i}}\right)_{j_{i} \in \cup_{i} J_{i}}, F$ et $G$, le $\mathbb{Z}$-module $\left(\operatorname{Sym}^{2} T^{*}\right)^{\Gamma_{k^{\prime}}}$ est libre de base $F \cdot F, F \cdot G, G \cdot G,\left(F \cdot D_{i}\right)_{i \in I},\left(G \cdot D_{i}\right)_{i \in I}$ et $\left(D_{S}\right)_{S \in \cup_{i, i^{\prime} \in I} O_{i, i^{\prime}}}$.

D'après le Lemme 5.4, pour chaque $i$ avec $\left|J_{i}\right|$ pair, on choisit une orbite $S_{i}^{0} \in O_{i, i}$ telle que $\sigma\left(S_{i}^{0}\right)=S_{i}^{0}$ et $\left|S_{i}^{0}\right|=s_{i} \cdot\left|J_{i}\right| / 2$, où $s_{i}$ est un entier impair. S'il existe au moins un $i_{0}$ tel que $\left|J_{i_{0}}\right|$ est impair, on choisit une orbite $S_{i}^{1} \in O_{i_{0}, i}$ telle que $\sigma\left(S_{i}^{1}\right)=S_{i}^{1}$ pour chaque $i \neq i_{0}$ avec $\left|J_{i}\right|$ impair.

Dans la base de $\left(\operatorname{Sym}^{2} T^{*}\right)^{\Gamma_{k^{\prime}}}$, on peut remplacer $D_{S_{i}^{1}}$ par $D_{i_{0}} \cdot D_{i}$ (si $i_{0}$ existe), pour tous les $i \neq i_{0}$ avec $\left|J_{i}\right|$ impair. On définit $\left(A_{l}\right)_{l=1}^{6}$ :

$A_{1}$ est le $\mathbb{Z}$-module libre de base $F \cdot F,\left(F \cdot D_{i}\right)_{i \in I,\left|J_{i}\right|}$ impair et $\left(D_{i_{0}} \cdot D_{i}\right)_{i \neq i_{0},\left|J_{i}\right|} \mid$ impair (si $i_{0}$ existe).

$A_{2}$ est le $\mathbb{Z}$-module libre de base $\left(F \cdot D_{i}\right)_{i \in I,\left|J_{i}\right| \text { pair }}$ et $\left(D_{S_{i}^{0}}\right)_{i \in I,\left|J_{i}\right|}$ pair .

$A_{3}$ est le $\mathbb{Z}$-module libre de base $G \cdot F$.

$A_{4}$ est le $\mathbb{Z}$-module libre de base $\left(D_{S}\right)_{S \in O_{0}}$, où

$$
O_{0}:=\bigcup_{i, i^{\prime} \in I} O_{i, i^{\prime}} \backslash\left\{\left(\Delta_{i}\right)_{i},\left(S_{i}^{0}\right)_{\left|J_{i}\right|} \text { pair },\left(S_{i}^{1}\right)_{i \neq i_{0},\left|J_{i}\right| \text { impair }}\right\}
$$


$A_{5}$ est le $\mathbb{Z}$-module libre de base $\left(G \cdot D_{i}\right)_{i \in I}$ et $\left(D_{\Delta_{i}}\right)_{i \in I}$.

$A_{6}$ est le $\mathbb{Z}$-module libre de base $G \cdot G$.

On a $\left(\operatorname{Sym}^{2} T^{*}\right)^{\Gamma_{k^{\prime}}} \stackrel{\sim}{\rightarrow} \bigoplus_{l=1}^{6} A_{l}$. Puisque $G^{\prime}-G=\sum_{i} \sum_{j_{i}} D_{j_{i}}-\frac{\sum_{i}\left|J_{i}\right|}{2} F, D_{j_{i}}+D_{j_{i}}^{\prime}=F, \sigma\left(S_{i}^{0}\right)=S_{i}^{0}, \sigma\left(S_{i}^{1}\right)=S_{i}^{1}$, $\sigma\left(\Delta_{i}\right)=\Delta_{i}$ et $O_{0}$ est $\sigma$-invariant, on a que $\bigoplus_{l=1}^{l_{0}} A_{l} \subset\left(\operatorname{Sym}^{2} T^{*}\right)^{\Gamma_{k^{\prime}}}$ est $\operatorname{Gal}\left(k^{\prime} / k\right)$-invariant pour chaque $l_{0}=1, \ldots, 6$.

Pour tous les $1 \leq l \leq 6$, notons $A_{l_{0}}^{\prime}:=\bigoplus_{l=1}^{l_{0}} A_{l} / \bigoplus_{l=1}^{l_{0}-1} A_{l}$. Alors $A_{l_{0}}^{\prime}$ est un Gal $\left(k^{\prime} / k\right)$-module et

$$
A_{l_{0}} \hookrightarrow \bigoplus_{l=1}^{l_{0}} A_{l} \rightarrow A_{l_{0}}^{\prime}
$$

est un isomorphisme de groupes abéliens.

Il suffit de montrer que $H^{1}\left(\mathrm{Gal}\left(k^{\prime} / k\right), A_{l_{0}}^{\prime}\right)=0$ pour tous les $1 \leq l \leq 6$.

Pour $l_{0}=1$, si $i_{0}$ n'existe pas, on a $A_{1}=\mathbb{Z} \cdot(F \cdot F)$ et $H^{1}\left(\mathrm{Gal}\left(k^{\prime} / k\right), A_{1}\right)=0$.

Supposons que $i_{0}$ existe. Soit $B_{1}$ le groupe abélien engendré librement par $F \cdot D_{i_{0}}$ et $F \cdot D_{i_{0}}^{\prime}$. Ainsi $H^{1}\left(\mathrm{Gal}\left(k^{\prime} / k\right), B_{1}\right)=0$. Soit $B_{1}^{\prime}$ le sous-groupe de $A_{1}$ engendré librement par $\left|J_{i_{0}}\right| F \cdot F, F \cdot D_{i_{0}},\left|J_{i_{0}}\right| F \cdot D_{i}$ pour tous les $i \neq i_{0}$ avec $\left|J_{i}\right|$ impair et $D_{i_{0}} \cdot D_{i}$ pour tous les $i \neq i_{0}$ avec $\left|J_{i}\right|$ impair. Ainsi $\left|A_{1} / B_{1}^{\prime}\right|=\left|J_{i_{0}}\right|^{M}$, où $M$ est le nombre de $i \in I$ avec $J_{i}$ impair. Donc $\left|A_{1} / B_{1}^{\prime}\right|$ est impair. Puisque $F \cdot D_{i_{0}}^{\prime}=\left|J_{i_{0}}\right| F \cdot F-F \cdot D_{i_{0}}$, $B_{1}$ est un sous-groupe de $B_{1}^{\prime}$.

Notons $C_{1}:=B_{1}^{\prime} / B_{1}$. Ainsi $C_{1}$ est engendré librement par les classes de $\left|J_{i_{0}}\right| F \cdot D_{i}$ et de $D_{i_{0}} \cdot D_{i}$ pour tous les $i \neq i_{0}$ avec $\left|J_{i}\right|$ impair. On a

$$
\sigma\left(D_{i_{0}} \cdot D_{i}\right)=D_{i_{0}}^{\prime} \cdot D_{i}^{\prime}=\left|J_{i}\right| F \cdot D_{i_{0}}^{\prime}-\left|J_{i_{0}}\right| F \cdot D_{i}+D_{i_{0}} \cdot D_{i} .
$$

Ainsi $C_{1}$ est engendré librement par $D_{i_{0}} \cdot D_{i}$ et $\sigma\left(D_{i_{0}} \cdot D_{i}\right)$ pour tous les $i \neq i_{0}$ avec $\left|J_{i}\right|$ impair. Donc $C_{1}$ est $\operatorname{Gal}\left(k^{\prime} / k\right)$ invariant, et $H^{1}\left(\operatorname{Gal}\left(k^{\prime} / k\right), C_{1}\right)=0$.

Puisque $H^{1}\left(\operatorname{Gal}\left(k^{\prime} / k\right), B_{1}\right)=0$, on a $B_{1}^{\prime}$ est $\operatorname{Gal}\left(k^{\prime} / k\right)$ invariant, et $H^{1}\left(\operatorname{Gal}\left(k^{\prime} / k\right), B_{1}^{\prime}\right)=0$. Donc $A_{1} / B_{1}^{\prime}$ est $\operatorname{Gal}\left(k^{\prime} / k\right)$ invariant, et $H^{1}\left(\mathrm{Gal}\left(k^{\prime} / k\right), A_{1} / B_{1}^{\prime}\right)=0$, parce que $\left|A_{1} / B_{1}^{\prime}\right|$ est impair. Donc $A_{1}$ est $\mathrm{Gal}\left(k^{\prime} / k\right)$ invariant, et $H^{1}\left(\mathrm{Gal}\left(k^{\prime} / k\right), A_{1}\right)=0$.

Pour $l_{0}=2$, soit $B_{2}$ un $\mathbb{Z}$-module libre de base $\left(\sigma\left(D_{S_{i}^{0}}\right)\right)_{i \in I,\left|J_{i}\right| \text { pair }}$ et $\left(D_{S_{i}^{0}}\right)_{i \in I,\left|J_{i}\right|}$ pair. On a $H^{1}\left(\operatorname{Gal}\left(k^{\prime} / k\right), B_{2}\right)=0$. On a

$$
\sigma\left(D_{S_{i}^{0}}\right)-D_{S_{i}^{0}}=\sum_{\left(j_{i}, j_{i^{\prime}}\right) \in S_{i}^{0}}\left(\left(F-D_{j_{i}}\right) \cdot\left(F-D_{j_{i^{\prime}}}\right)-D_{j_{i}} \cdot D_{j_{i^{\prime}}}\right)=\left|S_{i}^{0}\right|^{2} F \cdot F-\sum_{\left(j_{i}, j_{i^{\prime}}\right) \in S_{i}^{0}}\left(D_{j_{i}}+D_{j_{i^{\prime}}}\right) \cdot F .
$$

Puisque $\sigma\left(D_{S_{i}^{0}}\right)-D_{S_{i}^{0}}$ est $\Gamma_{k^{\prime}}$-invariant, $\sum_{\left(j_{i}, j_{i^{\prime}}\right) \in S_{i}^{0}}\left(D_{j_{i}}+D_{j_{i^{\prime}}}\right)$ est $\Gamma_{k^{\prime}}$-invariant et donc

$$
\sigma\left(D_{S_{i}^{0}}\right)-D_{S_{i}^{0}}=\left|S_{i}^{0}\right|^{2} F \cdot F-s_{i} F \cdot D_{i}
$$

Alors $\operatorname{Im}\left(B_{2} \subset\left(A_{1} \oplus A_{2}\right) \rightarrow A_{2}^{\prime}\right)$ est le sous-groupe engendré par l'élément $\left(s_{i} F \cdot D_{i}\right)_{i \in I,\left|J_{i}\right| \text { pair }}$ et $\left(D_{S_{i}^{0}}\right)_{i \in I,\left|J_{i}\right|}$ pair. D'après (5.1), la composition $B_{2} \subset\left(A_{1} \oplus A_{2}\right) \rightarrow A_{2}^{\prime}$ est injective, et $\left|A_{2}^{\prime} / B_{2}\right|=\prod_{i \in I,\left|J_{i}\right|}$ pair $s_{i}$. Ainsi $\left|A_{2}^{\prime} / B_{2}\right|$ est impair, et donc $H^{1}\left(\operatorname{Gal}\left(k^{\prime} / k\right), A_{2}^{\prime} / B_{2}\right)=0$. Ainsi $H^{1}\left(\operatorname{Gal}\left(k^{\prime} / k\right), A_{2}^{\prime}\right)=0$.

Pour $l_{0}=3$, on a

$$
\sigma(F \cdot G)=F \cdot G^{\prime}=F \cdot G+\sum_{i} F \cdot D_{i}-\frac{\sum_{i}\left|J_{i}\right|}{2} F \cdot F .
$$

Donc $(\sigma(F \cdot G)-F \cdot G) \in A_{1} \oplus A_{2}$. Alors l'action de $\operatorname{Gal}\left(k^{\prime} / k\right)$ sur $A_{3}^{\prime}$ est triviale, et donc $H^{1}\left(\mathrm{Gal}\left(k^{\prime} / k\right), A_{3}^{\prime}\right)=$ 0 .

Soit $(F \cdot E) \in\left(\operatorname{Sym}^{2} T^{*}\right)^{\Gamma_{k^{\prime}}}$, où $E \in T^{*}$. Alors $E \in\left(T^{*}\right)^{\Gamma_{k^{\prime}}}$. Ainsi $E$ est une combinaison de $G, F$ et $\left(D_{i}\right)_{i \in I}$, alors $(F \cdot E) \in A_{1} \oplus A_{2} \oplus A_{3}$. 
Pour $l_{0}=4$, on a $\left(D_{j_{i}}^{\prime} \cdot D_{j_{i^{\prime}}}^{\prime}\right)=D_{j_{i}} \cdot D_{j_{i^{\prime}}}+F \cdot\left(F-D_{j_{i}}-D_{j_{i^{\prime}}}\right)$ et donc pour chaque orbite $S,\left(\sigma\left(D_{S}\right)-D_{\sigma(S)}\right) \in$ $A_{1} \oplus A_{2} \oplus A_{3}$. Ainsi dans $\left(\oplus_{l=1}^{4} A_{l}\right) /\left(\oplus_{l=1}^{3} A_{l}\right)$, on a $\sigma\left(D_{S}\right)=D_{\sigma(S)}$. Donc l'action de $\operatorname{Gal}\left(k^{\prime} / k\right)$ sur $A_{4}^{\prime}$ est une permutation, et $H^{1}\left(\operatorname{Gal}\left(k^{\prime} / k\right), A_{4}^{\prime}\right)=0$.

Pour $l_{0}=5$, en utilisant $G^{\prime}-G=\sum_{i} \sum_{j_{i}} D_{j_{i}}-\frac{\sum_{i}\left|J_{i}\right|}{2} F$, on trouve

$$
\left(G^{\prime} \cdot D_{i}-D_{\Delta_{i}}-G \cdot D_{i}\right) \in A_{1} \oplus A_{2} \oplus A_{4} .
$$

Ainsi $A_{5}^{\prime}$ est engendré librement par $G^{\prime} \cdot D_{i}$ et $G \cdot D_{i}$ pour tous les $i \in I$. On a

$$
\sigma\left(G^{\prime} \cdot D_{i}\right)=G \cdot D_{i}^{\prime}=-G \cdot D_{i}+\left|J_{i}\right| G \cdot F .
$$

Donc dans $A_{5}^{\prime}$, on a $\sigma\left(G^{\prime} \cdot D_{i}\right)=-G \cdot D_{i}$. Donc $H^{1}\left(G a l\left(k^{\prime} / k\right), A_{5}^{\prime}\right)=0$.

Pour $l_{0}=6$, en utilisant $G^{\prime}-G=\sum_{i} \sum_{j_{i}} D_{j_{i}}-\frac{\sum_{i}\left|J_{i}\right|}{2} F$, on a

$$
(\sigma(G \cdot G)-G \cdot G) \in \bigoplus_{l=1}^{5} A_{l} .
$$

Donc dans $A_{6}^{\prime}, \sigma(G \cdot G)=G \cdot G$, et $H^{1}\left(\operatorname{Gal}\left(k^{\prime} / k\right), A_{6}^{\prime}\right)=0$.

\section{Surfaces de del Pezzo}

Dans cette section, soient $X$ une surface de del Pezzo sur $k$ et $\mathcal{T}$ un torseur universel de $X$. On cherche à conrôler l'exposant de torsion du quotient $\frac{H_{\mathrm{nr}}^{3}(\mathcal{T}, \mathbb{Q} / \mathbb{Z}(2))}{H^{3}(k, \mathbb{Q} / \mathbb{Z}(2))}$.

Une surface projective, lisse, géométriquement connexe $X$ est appelée surface de del Pezzo si le faisceau anticanonique $-K_{X}$ est ample. Le degré d'une telle surface $X$ est $\operatorname{deg}(X):=\left(K_{X}, K_{X}\right)$. Par [Koll96, Exercise 3.9], $X$ est alors géométriquement rationnelle, on a $1 \leq \operatorname{deg}(X) \leq 9$ et $\operatorname{Pic}\left(X_{\bar{k}}\right) \stackrel{\sim}{\rightarrow} \mathbb{Z}^{10-\operatorname{deg}(X)}$.

Soit $X$ une surface de del Pezzo de degré $d \leq 6$. Soit $r=9-d$. Rappelons des résultats dans [Ma, Chapter 4]. On sait ([Ma, Prop. 25.1]) qu'il existe une base $\left(l_{i}\right)_{i=0}^{r}$ de $\operatorname{Pic}\left(X_{\bar{k}}\right)$ telle que

$$
\left(l_{0}, l_{0}\right)=1, \quad\left(l_{i}, l_{i}\right)=-1 \text { si } i \neq 0, \quad\left(l_{i}, l_{j}\right)=0 \text { si } i \neq j \quad \text { et } \quad \omega=-3 l_{0}+\sum_{i=1}^{r} l_{i},
$$

où $\omega:=\left[K_{X}\right] \in \operatorname{Pic}\left(X_{\bar{k}}\right)$. Soit $\mathfrak{S}_{r}$ le groupe symétrique d'indice $r$. L'action de $\mathfrak{S}_{r}$ sur $\left\{l_{i}\right\}_{i=0}^{r}$, qui préserve $l_{0}$ et permute $\left(l_{i}\right)_{i=1}^{r}$, induit une action de permutation de $\mathfrak{S}_{r} \operatorname{sur} \operatorname{Pic}\left(X_{\bar{k}}\right)$. Pour $r=3,4,5,6,7,8$, soient $R_{r}$ le système de racines de type $A_{1} \times A_{2}, A_{4}, D_{5}, E_{6}, E_{7}, E_{8}$ respectivement. Par [Ma, Thm. 23.9], on a :

(1) le groupe de Weyl $W\left(R_{r}\right)$ agit sur $\operatorname{Pic}\left(X_{\bar{k}}\right)$ et cette action préserve $\omega$ et l'intersection;

(2) l'action de $\Gamma_{k}$ sur $\operatorname{Pic}\left(X_{\bar{k}}\right)$, qui préserve $\omega$ et l'intersection, se factorise par le groupe de Weyl $W\left(R_{r}\right)$;

(3) l'action de $\mathfrak{S}_{r}$ sur $\operatorname{Pic}\left(X_{\bar{k}}\right)$ ci-dessus, qui préserve $\omega$ et l'intersection aussi, induit une inclusion canonique $\mathfrak{S}_{r} \subset W\left(R_{r}\right)$ ne dépendant que du choix de la base $\left(l_{i}\right)_{i=0}^{r}$.

Proposition 6.1. Soient $X$ une surface de del Pezzo de degré $\geq 5$ et $\mathcal{T}$ un torseur universel de $X$. Alors $\frac{H_{\mathrm{nr}}^{3}(\mathcal{T}, \mathbb{Q} / \mathbb{Z}(2))}{H^{3}(k, \mathbb{Q} / \mathbb{Z}(2))}=0$.

Démonstration. Par un résultat classique (cf. [Cao, Lem. 3.2]), le $\Gamma_{k}$-module $\operatorname{Pic}\left(X_{\bar{k}}\right)$ est stablemement de permutation. D'après $(1.2)$, on a $H^{1}\left(k, \operatorname{Sym}^{2} \operatorname{Pic}\left(X_{\bar{k}}\right)\right)=0$. Par le théorème 4.7 et sa preuve, il suffit de montrer que l'on a $X(k) \neq \emptyset$ ou que le morphisme $\left(\operatorname{Sym}^{2} T^{*}\right)^{\Gamma_{k}} \stackrel{\cup}{\rightarrow} \mathrm{CH}^{2}\left(X_{\bar{k}}\right) \cong \mathbb{Z}$ dans le théorème 4.7 (2) est surjectif.

Notons $d$ le degré de $X$.

Si $d=5$ ou 7, par les travaux de Enriques, Châtelet, Manin, Swinnerton-Dyer (voir [CT99, Section 4] ou [VA, Théorème 2.1]), on a $X(k) \neq \emptyset$.

Si $d=9$, on a $\operatorname{Pic}\left(X_{\bar{k}}\right)^{\Gamma_{k}}=\operatorname{Pic}\left(X_{\bar{k}}\right)$. Puisque $X_{\bar{k}} \cong \mathbb{P}_{\bar{k}}^{2}$, l'homomorphisme $\cup$ est surjectif.

Si $d=8$, on a l'une des possibilités suivantes (voir par exemple [AB, Exemples 3.1.3 et 3.1.4]) : 
(i) $X$ est un éclatement de $\mathbb{P}_{k}^{2}$ en un $k$-point, et dans ce cas, $X(k) \neq \emptyset$.

(ii) Il existe des coniques lisses $C_{1}, C_{2}$ sur $k$ telles que $X \stackrel{\sim}{\rightarrow} C_{1} \times C_{2}$. Dans ce cas, on a $\operatorname{Pic}\left(X_{\bar{k}}\right)^{\Gamma_{k}}=$ $\operatorname{Pic}\left(X_{\bar{k}}\right), X_{\bar{k}} \cong \mathbb{P}_{\bar{k}}^{1} \times \mathbb{P}_{\bar{k}}^{1}$ et donc l'homomorphisme $\cup$ est surjectif.

(iii) Il existe une extension de corps $K / k$ de degré 2 et une conique $C$ sur $K$ telles que $X \stackrel{\sim}{\rightarrow} R_{K / k} C$, où $R_{K / k}$ désigne la restriction à la Weil de $K$ à $k$. Dans ce cas, il existe $D_{1}, D_{2} \in \operatorname{Pic}\left(X_{\bar{k}}\right)$ tels que $\Gamma_{k}$ permute $D_{1}, D_{2}$ et l'intersection de $D_{1}$ et $D_{2}$ est un point. Alors $D_{1} \cdot D_{2} \in\left(\operatorname{Sym}{ }^{2} \operatorname{Pic}\left(X_{\bar{k}}\right)\right)^{\Gamma_{k}}$, $\cup\left(D_{1} \cdot D_{2}\right)=1$ et donc l'homomorphisme $\cup$ est surjectif.

Si $d=6$, avec les notations ci-dessus, soit (cf. [Ma, §25.5.7])

$$
\sigma: \operatorname{Pic}\left(X_{\bar{k}}\right) \rightarrow \operatorname{Pic}\left(X_{\bar{k}}\right): \sum_{i=0}^{3} a_{i} l_{i} \mapsto\left(a_{0}+c\right) l_{0}+\sum_{i=1}^{3}\left(a_{i}-c\right) l_{i} \quad \text { avec } \quad c:=a_{0}+a_{1}+a_{2}+a_{3},
$$

et on a $W\left(R_{3}\right) \cong \mathfrak{S}_{3} \times \mathbb{Z} / 2 \cdot \sigma$. Soit $L_{1}:=\sum_{1 \leq i<j \leq 3}\left(l_{0}-l_{i}\right) \cdot\left(l_{0}-l_{j}\right)$ et $L_{2}:=l_{0} \cdot\left(\omega+l_{0}\right)$. Puisque l'action de $\Gamma_{k}$ se factorise par $W\left(R_{3}\right)$, on peut calculer que $L_{1}, L_{2} \in\left(\operatorname{Sym}^{2} \operatorname{Pic}\left(X_{\bar{k}}\right)\right)^{\Gamma_{k}}$ et $\cup\left(L_{1}\right)=3, \cup\left(L_{2}\right)=-2$. Alors $\cup$ est surjectif.

Lemme 6.2. Soient $X$ une surface de del Pezzo de degré $d=1,2,3,4$ et $\mathcal{T}$ un torseur universel de X. Alors

$$
\frac{H_{\mathrm{nr}}^{3}(\mathcal{T}, \mathbb{Q} / \mathbb{Z}(2))}{H^{3}(k, \mathbb{Q} / \mathbb{Z}(2))}[p]=0 \text { pour tout nombre premier } \begin{cases}p \neq 2 & \text { si } d=4 \\ p \neq 2,3 & \text { si } d=2,3 \\ p \neq 2,3,5 & \text { si } d=1\end{cases}
$$

Démonstration. Soit $r=9-d$. Avec les notations ci-dessus, d'après [Ma, §26.6], \#W( $\left.R_{r}\right)=2^{7} 3 \cdot 5,2^{7} 3^{4} 5$, $2^{10} 3^{4} 5 \cdot 7,2^{14} 3^{5} 5^{2} 7$ pour $d=4,3,2,1$ respectivement. Alors, pour tout nombre premier $p$ de l'énoncé, tout $p$-groupe de Sylow de $\mathfrak{S}_{r}$ est un $p$-groupe de Sylow de $W\left(R_{r}\right)$. Dans ce cas, l'action de tout $p$-sous-groupe de $W\left(R_{r}\right)$ sur $\operatorname{Pic}\left(X_{\bar{k}}\right)$ est donc de permutation. C'est donc aussi le cas sur $\operatorname{Sym}^{2} \operatorname{Pic}\left(X_{\bar{k}}\right)$. Par un argument de corestriction-restriction, $H^{1}\left(k, \operatorname{Sym}^{2} \operatorname{Pic}\left(X_{\bar{k}}\right)\right)[p]=0$.

On considère le morphisme dans le Théorème $4.7(2):\left(\operatorname{Sym}^{2} T^{*}\right)^{\Gamma_{k}} \stackrel{\cup}{\rightarrow} \mathrm{CH}^{2}\left(X_{\bar{k}}\right) \cong \mathbb{Z}$. Par la définition, $\cup(\omega \cdot \omega)=4,3,2,1$ pour $d=4,3,2,1$ respectivement. On conclut alors avec le Théorème 4.7 (2).

Remarque 6.3. Sous les hypothèses du Lemme 6.2, par la même méthode, on peut montrer le résultat ci-dessous (qui est déjà connu) :

$$
\frac{\operatorname{Br}(X)}{\operatorname{Br}(k)}[p]=0 \text { pour tout nombre premier } \begin{cases}p \neq 2 & \text { si } d=4 \\ p \neq 2,3 & \text { si } d=2,3 \\ p \neq 2,3,5 & \text { si } d=1\end{cases}
$$

Théorème 6.4. Soient $X$ une surface de del Pezzo de degré 3 ou 4 et $\mathcal{T}$ un torseur universel de X. Alors $\frac{H_{\mathrm{nr}}^{3}(\mathcal{T}, \mathbb{Q} / \mathbb{Z}(2))}{H^{3}(k, \mathbb{Q} / \mathbb{Z}(2))}[p]=0$ pour tout $p \neq 2$.

Démonstration. D'après le Lemme 6.2 , il suffit de montrer que $\frac{H_{\mathrm{nr}}^{3}(\mathcal{T}, \mathbb{Q} / \mathbb{Z}(2))}{H^{3}(k, \mathbb{Q} / \mathbb{Z}(2))}[3]=0$ pour toute surface de del Pezzo $X$ de degré 3.

On suppose donc que le degré de $X$ est 3.

Notons $T^{*}:=\operatorname{Pic}\left(X_{\bar{k}}\right)$. Soient $\left(l_{i}\right)_{i=0}^{6}$ une base dans (6.1) et $\omega$ le fibré en droites canonique de $X$. Il y a 27 courbes exceptionnelles (les 27 droites) sur $X_{\bar{k}}$. Le groupe $\Gamma_{k}$ agit par permutation de ces droites. On les note $\left(D_{i}\right)_{i=1}^{27}$. D'après [Ma, prop. 26.1], on peut supposer $D_{i}=l_{i}$ pour $1 \leq i \leq 6, D_{i}=2 l_{0}-\sum_{j \neq i-6} l_{j}$ pour $7 \leq i \leq 12$ et $\left(D_{i}\right)_{i=13}^{27}=\left(l_{0}-l_{i}-l_{j}\right)_{1 \leq i<j \leq 6}$. 
Dans $\operatorname{Sym}^{2} T^{*}$, on note :

$$
L:=\left(l_{0} \cdot l_{0}\right)-\sum_{i=1}^{6}\left(l_{i} \cdot l_{i}\right),
$$

et on peut calculer que $6 L=5(\omega \cdot \omega)-\sum_{i=1}^{27}\left(D_{i} \cdot D_{i}\right)$. Donc l'action de $\Gamma_{k}$ sur $L$ est triviale.

On considère le morphisme dans le Théorème $4.7(2):\left(\operatorname{Sym}^{2} T^{*}\right)^{\Gamma_{k}} \stackrel{\cup}{\rightarrow} \mathrm{CH}^{2}\left(X_{\bar{k}}\right) \cong \mathbb{Z}$. Puisque $\cup(\omega$. $\omega)=3$ et $\cup(L)=7$, le morphisme $\cup$ est surjectif. Par le Théorème 4.7 (ii), il suffit de montrer que $H^{1}\left(k, \operatorname{Sym}^{2} T^{*}\right)[3]=0$.

Il existe une matrice $A \in M_{28 \times 28}(\mathbb{Z})$, telle que dans le réseau $S_{y m^{2}} T^{*}$, de rang 28 , on ait l'égalité de vecteurs :

$$
\left[-L,\left(D_{i} \cdot D_{i}\right)_{i=1}^{27}\right]^{t}=A \cdot\left[\left(l_{i} \cdot l_{i}\right)_{i=0}^{6},\left(-l_{0} \cdot l_{i}\right)_{i=1}^{6},\left(l_{i} \cdot l_{j}\right)_{1 \leq i<j \leq 6}\right]^{t} .
$$

Un calcul direct donne : $A=$

$$
\begin{aligned}
& {\left[\begin{array}{llllllllllllllllllllllllllll}
-1 & 1 & 1 & 1 & 1 & 1 & 1 & 0 & 0 & 0 & 0 & 0 & 0 & 0 & 0 & 0 & 0 & 0 & 0 & 0 & 0 & 0 & 0 & 0 & 0 & 0 & 0 & 0
\end{array}\right.} \\
& \begin{array}{llllllllllllllllllllllllllll}
0 & 1 & 0 & 0 & 0 & 0 & 0 & 0 & 0 & 0 & 0 & 0 & 0 & 0 & 0 & 0 & 0 & 0 & 0 & 0 & 0 & 0 & 0 & 0 & 0 & 0 & 0 & 0
\end{array} \\
& \begin{array}{llllllllllllllllllllllllllll}
0 & 0 & 1 & 0 & 0 & 0 & 0 & 0 & 0 & 0 & 0 & 0 & 0 & 0 & 0 & 0 & 0 & 0 & 0 & 0 & 0 & 0 & 0 & 0 & 0 & 0 & 0 & 0
\end{array} \\
& \begin{array}{llllllllllllllllllllllllllll}
0 & 0 & 0 & 1 & 0 & 0 & 0 & 0 & 0 & 0 & 0 & 0 & 0 & 0 & 0 & 0 & 0 & 0 & 0 & 0 & 0 & 0 & 0 & 0 & 0 & 0 & 0 & 0
\end{array} \\
& \begin{array}{llllllllllllllllllllllllllll}
0 & 0 & 0 & 0 & 1 & 0 & 0 & 0 & 0 & 0 & 0 & 0 & 0 & 0 & 0 & 0 & 0 & 0 & 0 & 0 & 0 & 0 & 0 & 0 & 0 & 0 & 0 & 0
\end{array} \\
& \begin{array}{llllllllllllllllllllllllllll}
0 & 0 & 0 & 0 & 0 & 1 & 0 & 0 & 0 & 0 & 0 & 0 & 0 & 0 & 0 & 0 & 0 & 0 & 0 & 0 & 0 & 0 & 0 & 0 & 0 & 0 & 0 & 0
\end{array} \\
& \begin{array}{llllllllllllllllllllllllllll}
0 & 0 & 0 & 0 & 0 & 0 & 1 & 0 & 0 & 0 & 0 & 0 & 0 & 0 & 0 & 0 & 0 & 0 & 0 & 0 & 0 & 0 & 0 & 0 & 0 & 0 & 0 & 0
\end{array} \\
& \begin{array}{llllllllllllllllllllllllllll}
4 & 0 & 1 & 1 & 1 & 1 & 1 & 0 & 4 & 4 & 4 & 4 & 4 & 0 & 0 & 0 & 0 & 0 & 2 & 2 & 2 & 2 & 2 & 2 & 2 & 2 & 2 & 2
\end{array} \\
& \begin{array}{llllllllllllllllllllllllllll}
4 & 1 & 0 & 1 & 1 & 1 & 1 & 4 & 0 & 4 & 4 & 4 & 4 & 0 & 2 & 2 & 2 & 2 & 0 & 0 & 0 & 0 & 2 & 2 & 2 & 2 & 2 & 2
\end{array} \\
& \begin{array}{llllllllllllllllllllllllllll}
4 & 1 & 1 & 0 & 1 & 1 & 1 & 4 & 4 & 0 & 4 & 4 & 4 & 2 & 0 & 2 & 2 & 2 & 0 & 2 & 2 & 2 & 0 & 0 & 0 & 2 & 2 & 2
\end{array} \\
& \begin{array}{llllllllllllllllllllllllllll}
4 & 1 & 1 & 1 & 0 & 1 & 1 & 4 & 4 & 4 & 0 & 4 & 4 & 2 & 2 & 0 & 2 & 2 & 2 & 0 & 2 & 2 & 0 & 2 & 2 & 0 & 0 & 2
\end{array} \\
& \begin{array}{llllllllllllllllllllllllllll}
4 & 1 & 1 & 1 & 1 & 0 & 1 & 4 & 4 & 4 & 4 & 0 & 4 & 2 & 2 & 2 & 0 & 2 & 2 & 2 & 0 & 2 & 2 & 0 & 2 & 0 & 2 & 0
\end{array} \\
& \begin{array}{llllllllllllllllllllllllllll}
4 & 1 & 1 & 1 & 1 & 1 & 0 & 4 & 4 & 4 & 4 & 4 & 0 & 2 & 2 & 2 & 2 & 0 & 2 & 2 & 2 & 0 & 2 & 2 & 0 & 2 & 0 & 0
\end{array} \\
& \begin{array}{llllllllllllllllllllllllllll}
1 & 1 & 1 & 0 & 0 & 0 & 0 & 2 & 2 & 0 & 0 & 0 & 0 & 2 & 0 & 0 & 0 & 0 & 0 & 0 & 0 & 0 & 0 & 0 & 0 & 0 & 0 & 0
\end{array} \\
& \begin{array}{llllllllllllllllllllllllllll}
1 & 1 & 0 & 1 & 0 & 0 & 0 & 2 & 0 & 2 & 0 & 0 & 0 & 0 & 2 & 0 & 0 & 0 & 0 & 0 & 0 & 0 & 0 & 0 & 0 & 0 & 0 & 0
\end{array} \\
& \begin{array}{llllllllllllllllllllllllllll}
1 & 1 & 0 & 0 & 1 & 0 & 0 & 2 & 0 & 0 & 2 & 0 & 0 & 0 & 0 & 2 & 0 & 0 & 0 & 0 & 0 & 0 & 0 & 0 & 0 & 0 & 0 & 0
\end{array} \\
& \begin{array}{llllllllllllllllllllllllllll}
1 & 1 & 0 & 0 & 0 & 1 & 0 & 2 & 0 & 0 & 0 & 2 & 0 & 0 & 0 & 0 & 2 & 0 & 0 & 0 & 0 & 0 & 0 & 0 & 0 & 0 & 0 & 0
\end{array}
\end{aligned}
$$

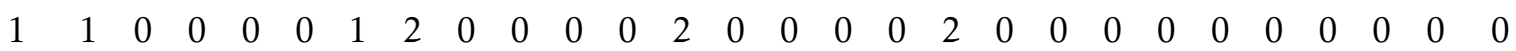

$$
\begin{aligned}
& \begin{array}{llllllllllllllllllllllllllll}
1 & 0 & 1 & 1 & 0 & 0 & 0 & 0 & 2 & 2 & 0 & 0 & 0 & 0 & 0 & 0 & 0 & 0 & 2 & 0 & 0 & 0 & 0 & 0 & 0 & 0 & 0 & 0
\end{array}
\end{aligned}
$$

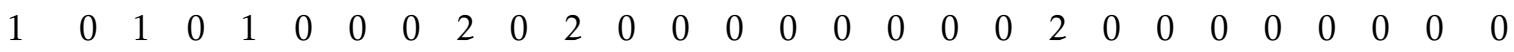

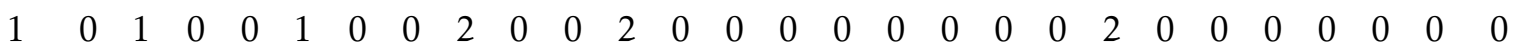

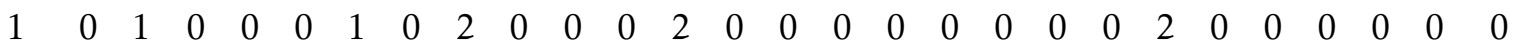

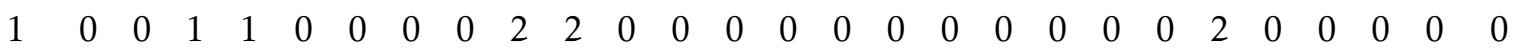

$$
\begin{aligned}
& \begin{array}{llllllllllllllllllllllllllll}
1 & 0 & 0 & 1 & 0 & 1 & 0 & 0 & 0 & 2 & 0 & 2 & 0 & 0 & 0 & 0 & 0 & 0 & 0 & 0 & 0 & 0 & 0 & 2 & 0 & 0 & 0 & 0
\end{array}
\end{aligned}
$$

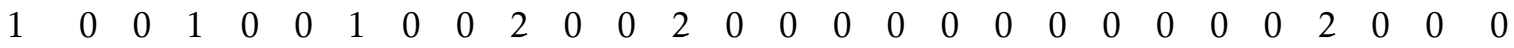

$$
\begin{aligned}
& 1 \quad 0 \begin{array}{lllllllllllllllllllllllllll} 
& 0 & 0 & 1 & 1 & 0 & 0 & 0 & 0 & 2 & 2 & 0 & 0 & 0 & 0 & 0 & 0 & 0 & 0 & 0 & 0 & 0 & 0 & 0 & 2 & 0 & 0
\end{array}
\end{aligned}
$$

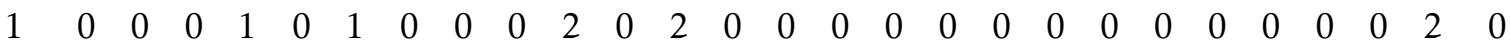

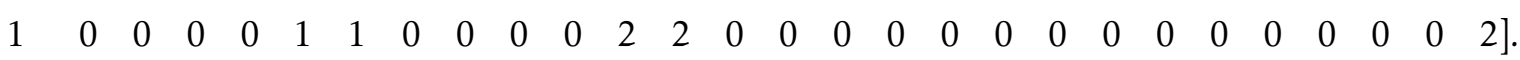

Par calcul direct (et aussi par ordinateur) on établit $\operatorname{det}(A)=5 \cdot 2^{27}$. D'après $(6.2)$, le $\mathbb{Z}_{3}$-module $\left(\operatorname{Sym}^{2} T^{*}\right) \otimes$ $\mathbb{Z}_{3}$ est libre avec une base $:-L,\left(D_{i} \cdot D_{i}\right)_{i=1}^{27}$. Puisque le groupe $\Gamma_{k}$ agit par permutation des vecteurs 
$-L,\left(D_{i} \cdot D_{i}\right)_{i=1}^{27}$, on a

$$
H^{1}\left(k,\left(\operatorname{Sym}^{2} T^{*}\right) \otimes \mathbb{Z}_{3}\right) \cong 0 \quad \text { et } \quad H^{1}\left(k, \operatorname{Sym}^{2} T^{*}\right)[3]=0 .
$$

Corollaire 6.5. Soient $X$ une surface de del Pezzo de degré 2. $\mathcal{T}$ un torseur universel de $X$ et $\mathcal{T}^{c}$ une compactification lisse de $\mathcal{T}$. Alors $\frac{H_{\mathrm{nr}}^{3}\left(\mathcal{T}^{c}, \mathbb{Q} / \mathbb{Z}(2)\right)}{H^{3}(k, \mathbb{Q} / \mathbb{Z}(2))}[p]=0$ pour tout $p \neq 2$.

Démonstration. D'après le Lemme 6.2, il suffit de montrer $\frac{H_{\mathrm{nr}}^{3}\left(\mathcal{T}^{c}, \mathbb{Q} / \mathbb{Z}(2)\right)}{H^{3}(k, \mathbb{Q} / \mathbb{Z}(2))}[3]=0$.

Sous les notations ci-dessus, on a l'inclusion canonique $W\left(R_{6}\right) \subset W\left(R_{7}\right)$ et les sous- $p$-groupes de Sylow de $W\left(R_{6}\right)$ et de $W\left(R_{7}\right)$ sont isomorphes pour tout $p \neq 2$.

Dans le cas d'une surface de del Pezzo de degré 2, l'action de $\Gamma_{k} \operatorname{sur} \operatorname{Pic}\left(X_{\bar{k}}\right)$ se factorise par $W\left(R_{7}\right)$, et donc, après conjugaison, il existe une extension de corps $k \subset k^{\prime}$ avec $\left(\left[k^{\prime}: k\right], p\right)=1$ telle que l'action de $\Gamma_{k^{\prime}}$ sur $\operatorname{Pic}\left(X_{\bar{k}}\right)$ se factorise par $W\left(R_{6}\right) \subset W\left(R_{7}\right)$. Par [Ma, Cor. 26.7], dans $X_{k^{\prime}}$, il existe une courbe exceptionnelle définie sur $k^{\prime}$, donc il existe une surface de del Pezzo de degré $3 X^{\prime}$ sur $k^{\prime}$ et un $k^{\prime}$-morphisme propre, surjectif, birationnel $X_{k^{\prime}} \rightarrow X^{\prime}$. D'après le Théorème 6.4 et un argument de restriction-inflation, on a $\frac{H_{\mathrm{nr}}^{3}\left(\mathcal{T}^{c}, \mathbb{Q} / \mathbb{Z}(2)\right)}{H^{3}(k, \mathbb{Q} / \mathbb{Z}(2))}[3]=0$.

Example 6.6. Soit $k$ un corps contenant une racine cubique de l'unité non-triviale. Soit $X$ une surface cubique diagonale d'équation :

$$
a x^{3}+b y^{3}+c z^{3}+d t^{3}=0
$$

en les variables $x, y, z, t$, avec $a, b, c, d \in k^{\times}$. Soit $K:=k(\sqrt[3]{b / a}, \sqrt[3]{a d / b c})$. Alors $X_{K}$ est une $K$-surface $K$ rationnelle. D'après le Corollaire 2.10, $\frac{H_{\mathrm{nr}}^{3}\left(\mathcal{T}^{c}, \mathbb{Q} / \mathbb{Z}(2)\right)}{H^{3}(k, \mathbb{Q} / \mathbb{Z}(2))}$ est annulé par 9. D'après le Théorème 6.4, on a $\frac{H_{\mathrm{nr}}^{3}\left(\mathcal{T}^{c}, \mathbb{Q} / \mathbb{Z}(2)\right)}{H^{3}(k, \mathbb{Q} / \mathbb{Z}(2))}=0$.

Théorème 6.7. Soit $X$ une $k$-surface projective, lisse, géométriquement rationnelle. Soit $\mathcal{T} \rightarrow X$ un torseur universel sur $X$ et soit $\mathcal{T}^{c}$ une $k$-compactification lisse de $\mathcal{T}$. Supposons que la surface $X$ est $k$-birationnellement équivalente à une surface de del Pezzo de degré $\geq 2$ ou à une surface fibrée en coniques au-dessus d'une conique. Alors $\frac{H_{\mathrm{nr}}^{3}\left(\mathcal{T}^{c}, \mathbb{Q} / \mathbb{Z}(2)\right)}{H^{3}(k, \mathbb{Q} / \mathbb{Z}(2))}[p]=0$ pour tout $p \neq 2$.

Démonstration. Soit $X$ une surface fibrée en coniques au-dessus d'une conique $C$. Il existe une extension $K_{1} / k$ de degré 2 telle que $C_{K_{1}} \cong \mathbb{P}_{K_{1}}^{1}$. Notons $\eta$ le point générique de $C$ et $X_{\eta}$ sa fibre. Alors $X_{\eta}$ est une conique, et donc elle définit $\left[X_{\eta}\right] \in \operatorname{Br}(k(C))[2]$. Puisque $\operatorname{Br}(\bar{k}(C))=0$, il existe une extension finie galoisienne $k^{\prime} / k$ tel que $\left[X_{\eta}\right] \|_{k^{\prime}(C)}=0$. Soient et $k \subset K_{2} \subset k^{\prime}$ l'extension correspondant à un $p$-sous-groupe de Sylow de $\operatorname{Gal}\left(k^{\prime} / k\right)$. Alors $p \nmid\left[K_{2}: k\right]$ et, par un argument de restriction-inflation, $\left[X_{\eta}\right]_{K_{2}(C)}=0$. Soit $K:=K_{1} \cdot K_{2}$. On a $p \nmid[K: k], C_{K} \cong \mathbb{P}_{K}^{1}$ et $X_{\eta} \times_{k} K \cong \mathbb{P}_{K(C)}^{1}$. Donc $X_{K}$ est $K$-rationnelle. D'après le Corollaire 2.10, l'énoncé vaut pour une telle surface $X$.

Soit $X$ une surface de del Pezzo de degré $\geq 2$. D'après la Proposition 6.1, le Théorème 6.4 et le Corollaire 6.5 , l'énoncé vaut pour une telle surface $X$.

En général, soit $X_{1}$ une surface projective, lisse, géométriquement rationnelle telle que $X$ et $X_{1}$ soient birationnellement équivalents. Il existe un torseur universel $\mathcal{T}_{1}^{c}$ de $X_{1}$ tel que $\mathcal{T}^{c}$ et $\mathcal{T}_{1}^{c}$ soient stablement birationnellement équivalents par [CTS87, Prop. 2.9.2]. Donc $H_{\mathrm{nr}}^{3}\left(\mathcal{T}^{c}, \mathbb{Q} / \mathbb{Z}(2)\right) \stackrel{\sim}{\rightarrow} H_{\mathrm{nr}}^{3}\left(\mathcal{T}_{1}^{c}, \mathbb{Q} / \mathbb{Z}(2)\right)$ et on peut remplacer $X$ par $X_{1}$. Donc on peut supposer que $X$ est une surface de del Pezzo de degré $\geq 2$ ou une surface fibrée en coniques au-dessus d'une conique. On obtient le théorème en rassemblant les résultats ci-dessus.

Par la classification $k$-birationnelle des surfaces projectives, lisses (cf. [Koll96, Thm. 2.1]), toute surface projective, lisse, géométriquement rationnelle est $k$-birationnellement équivalent à soit une surface de del Pezzo, soit une surface fibrée en coniques au-dessus d'une conique. Dans le Théorème 6.7, le seul cas que l'on ne traite pas est alors le cas où $X$ est une surface de del Pezzo $k$-minimale de degré 1 . 


\section{Remerciements}

Nous remercions Jean-Louis Colliot-Thélène pour plusieurs discussions. Je remercie vivement les rapporteurs de l'Épijournal de Géométrie Algébrique pour leurs commentaires. Projet soutenu par l'attribution d'une allocation de recherche Région Ile-de-France.

\section{Références}

[AB] A. Auel et M. Bernardara, Semiorthogonal decompositions and birational geometry of del Pezzo surfaces over arbitrary fields, Proc. Lond. Math. Soc. (3) 117 (2018), no. 1, 1-64. MR-3830889

[An73] S. Anantharaman, Schémas en groupes, espaces homogènes et espaces alébriques sur une base de dimension 1. Dans : Sur les groupes algébriques, pp. 5-79, Bull. Soc. Math. France, Mém. 33, Soc. Math. France, Paris, 1973. MR-0335524

[BBD] A. Beilinson, J. Bernstein et P. Deligne, Faisceaux pervers. Dans : Analyse et topologie sur les espaces singuliers I (Luminy, 1981), pp. 5-171, Astérisque, vol. 100, Soc. Math. France (1982). MR-0751966

[Bour] N. Bourbaki, Éléments de mathématique. Algèbre. Chapitres 1 à 3, Hermann, Paris, 1970. MR-0274237

[Cao] Y. Cao, Cohomologie non ramifiée de degré 3 : variétés cellulaires et surfaces de del Pezzo de degré au moins 5, Ann. K-Theory 3 (2018), no. 1, 157-171. MR-3695367

[CX] Y. Cao et F. Xu, Strong approximation with Brauer-Manin obstruction for toric varieties, Ann. Inst. Fourier (Grenoble), à paraître. arXiv:1311.7655

[CT83] J.-L. Colliot-Thélène, Hilbert's theorem 90 for $K_{2}$, with application to the Chow groups of rational surfaces, Invent. math. 71 (1983), no. 1, 1-20. MR-0688259

[CT87] J.-L. Colliot-Thélène, Arithmétique des variétés rationnelles et problèmes birationnels. Dans : Proceedings of the International Congress of Mathematicians, vol. 1 (Berkeley, California, 1986), pp. 641-653, Amer. Math. Soc., Providence, RI, 1987. MR-0934267

[CT91] J.-L. Colliot-Thélène, Cycles algébriques de torsion et K-théorie algébrique. Dans : Arithmetic algebraic geometry (Trento, 1991), pp. 1-49, Lecture Notes in Math., vol. 1553, Springer, Berlin, 1993. MR1338859

[CT95] J.-L. Colliot-Thélène, Birational invariants, purity and the Gersten conjecture. Dans : K-Theory and algebraic geometry : connections with quadratic forms and division algebras (Santa Barbara, CA, 1992), pp. 1-64, Proc. Sympos. Pure Math., vol. 58, Part 1, Amer. Math. Soc., Providence, RI, 1995. MR-1327280

[CT99] J.-L. Colliot-Thélène, Points rationnels sur les variétés non de type général. Chapitre II : Surfaces rationnelles, 1999. Voir https://www.math.u-psud.fr/ colliot/Cours99_ChapII.pdf

[CT15] J.-L. Colliot-Thélène, Descente galoisienne sur le second groupe de Chow : mise au point et applications, Doc. Math. (2015), extra vol. : Alexander S. Merkurjev's sixtieth Birthday, 195-220. MR-3404380

[CTCS] J.-L. Colliot-Thélène, D. Coray et J.-J. Sansuc, Descente et principe de Hasse pour certaines variétés rationnelles, J. Reine Angew. Math. 320 (1980), 150-191. MR-0592151

[CTHK] J.-L. Colliot-Thélène, R. T. Hoobler et B. Kahn, The Bloch-Ogus-Gabber theorem. Dans : Algebraic K-theory (Toronto, ON, 1996), pp. 31-94, Fields Inst. Commun., vol. 16, Amer. Math. Soc., Providence, RI, 1997. MR-1466971

[CTHS] J.-L. Colliot-Thélène, D. Harari et A. N. Skorobogatov, Compactification équivariante d'un tore (d'après Brylinski et Künnemann), Expo. Math. 23 (2005), no. 2, 161-170. MR-2155008

[CTS77] J.-L. Colliot-Thélène et J.-J. Sansuc, Variétés de première descente attachées aux variétés rationnelles, C. R. Acad. Sci. Paris Sér. A-B 284 (1977), no. 16, A967-A970. MR-0447246 
[CTS80] J.-L. Colliot-Thélène et J.-J. Sansuc, La descente sur les variétés rationnelles. Dans : Journées de Géométrie Algébrique d'Angers (Juillet 1979), pp. 223-237, Sijthhof \& Noordhoff, Alphen aan den Rijn-Germantown, Md., 1980. MR-0605344

[CTS81] J.-L. Colliot-Thélène et J.-J. Sansuc, On the Chow groups of certain rational surfaces : a sequel to a paper of S. Bloch, Duke Math. J. 48 (1981), no. 2, 421-447. MR-0620258

[CTS87] J.-L. Colliot-Thélène et J.-J. Sansuc, La descente sur les variétés rationnelles II, Duke Math. J. 54 (1987), no. 2, 375-492. MR-0899402

[CTSSD] J.-L. Colliot-Thélène, J.-J. Sansuc et P. Swinnerton-Dyer, Intersections of two quadrics and Châtelet surfaces II, J. Reine Angew. Math. 374 (1987), 72-168. MR-0876222

[EKLV] H. Esnault, B. Kahn, M. Levine et E. Viehweg, The Arason invariant and mod 2 algebraic cycles, J. Amer. Math. Soc. 11 (1998), no. 1, 73-118. MR-1460391

[F] L. Fu, Étale cohomology theory, Nankai Tracts in Mathematics, vol. 13, World Scientific Publishing Co. Pte. Ltd., Hackensack, NJ, 2011. MR-2791606

[Fu84] W. Fulton, Intersection theory, Ergebnisse der Mathematik und ihrer Grenzgebiete (3), vol. 2, SpringerVerlag, Berlin, 1984. MR-0732620

[Fu93] W. Fulton, Introduction to toric varieties, Annals of Mathematics Studies, vol. 131, Princeton University Press, Princeton, NJ, 1993. MR-1234037

[HS] D. Harari et A. Skorobogatov, The Brauer group of torsors and its arithmetic applications, Ann. Inst. Fourier (Grenoble) 53 (2003), no. 7, 1987-2019. MR-2044165

[Ka93] B. Kahn, Descente galoisienne et $K_{2}$ des corps de nombres, K-theory 7 (1993), no. 1, 55-100. MR-1220427

[Ka96] B. Kahn, Applications of weight-two motivic cohomology, Doc. Math. 1 (1996), no. 17, 395-416. MR1423901

[Ka97] B. Kahn, Motivic cohomology of smooth geometrically cellular varieties. Dans : Algebraic K-theory (Seattle, WA, 1997), pp. 149-174, Proc. Symposia Pure Math., vol. 67, Amer. Math. Soc., Providence, RI, 1999. MR-1743239

[Koll96] J. Kollár, Rational curves on algebraic varieties, Ergebnisse der Mathematik und ihrer Grenzgebiete (3), vol. 32, Springer-Verlag, Berlin, 1996. MR-1440180

[KS] M. Kashiwara et P. Schapira, Categories and sheaves, Grundlehren der Mathematischen Wissenschaften, vol. 332, Springer-Verlag, Berlin, 2006. MR-2182076

[L87] S. Lichtenbaum, The construction of weight-two arithmetic cohomology, Invent. math. 88 (1987), no. 1, 183-215. MR-0877012

[L90] S. Lichtenbaum, New results on weight-two motivic cohomology. Dans : The Grothendieck Festschrift, Vol. III, pp. 35-55, Progr. Math., vol. 88, Birkhäuser Boston, Boston, MA, 1990. MR-1106910

[Ma] Y. Manin, Cubic forms. Algebra, geometry, arithmetic, 2nd edition, translated from the Russian by M. Hazewinkel, North-Holland Mathematical Library, vol. 4, North-Holland Publishing Co., Amsterdam, 1986. MR-0833513

[Me03] A. Merkurjev, Rost invariants of simply connected algebraic groups, with a section by S. Garibaldi. Dans : Cohomological Invariants in Galois Cohomology, pp. 101-158, Univ. Lecture Ser., vol. 28, Amer. Math. Soc., Providence, RI, 2003. MR-1999385

[Me08] A. Merkurjev, Unramified elements in cycle modules, J. London Math. Soc. (2) 78 (2008), no. 1, 51-64. MR-2427051

[Me16] A. Merkurjev, Weight two motivic cohomology of classifying spaces for semisimple groups, Amer. J. Math. 138 (2016), no. 3, 763-792. MR-3506385

[Oda] T. Oda, Convex bodies and algebraic geometry. An introduction to the theory of toric varieties. Ergebnisse der Mathematik und ihrer Grenzgebiete (3), vol. 15, Springer-Verlag, Berlin, 1988. MR-0922894 
[Pir] A. Pirutka, Sur le groupe de Chow de codimension deux des variétés sur les corps finis, Algebra Number Theory 5 (2011), no. 6, 803-817. MR-2923728

[PV] V. L. Popov et È. B. Vinberg, Invariant Theory. Dans : Algebraic geometry 4, pp. 137-314, 315, Itogi Nauki i Tekhniki, Sovrem. Probl. Mat. Fund. Naprav., vol. 55, Akad. Nauk SSSR, Vsesoyuz. Inst. Nauchn. i Tekhn. Inform., Moscow, 1989; traduction anglaise dans : Algebraic Geometry IV, pp. 123-278, Encyclopaedia of Mathematical Sciences, vol. 55, Springer-Verlag, Berlin, 1994. MR-1100485

[Ro96] M. Rost, Chow groups with coefficients, Doc. Math. 1 (1996), no. 16, 319-393. MR-1418952

[S] J.J. Sansuc, Groupe de Brauer et arithmétique des groupes algébriques linéaires sur un corps de nombres, J. reine angew. Math. 327, (1981), 12-80. MR-0631309

[Sko] A. Skorobogatov, Torsors and rational points, Cambridge Tracts in Mathematics, vol. 144, Cambridge University Press, Cambridge, 2001. MR-1845760

[Sum] H. Sumihiro, Equivariant completion, J. Math. Kyoto Univ. 14 (1974), 1-28. MR-0337963

[Su] A. A. Suslin, Torsion in $K_{2}$ of fields, K-Theory 1 (1987), no. 1, 5-29. MR-0899915

[VA] A. Várilly-Alvarado, Arithmetic of del Pezzo surfaces. Dans : Birational geometry, rational curves, and arithmetic, pp. 293-319, Simons Symp., Springer, Cham, 2013. MR-3114932 